\title{
Tendency to ruminate and anxiety are associated with altered alpha and beta oscillatory power dynamics during memory for contextual details
}

\author{
Nicole A. Forner-Phillips ${ }^{1}$ - Caitlin Mills ${ }^{1} \cdot$ Robert S. Ross ${ }^{1}$ \\ Published online: 19 May 2020 \\ (C) The Psychonomic Society, Inc. 2020
}

\begin{abstract}
Rumination occurs when an individual becomes mentally stuck and cannot redirect attention away from an unwanted thought demonstrating cognitive inflexibility. Cognitive flexibility is important for various cognitive functions, including episodic memory. Trait rumination is a partial mediator in the relationship between depression and overgeneral episodic memory, suggesting that rumination may negatively influence memory for contextual details. Oscillations in the alpha $(8-12 \mathrm{~Hz})$ and beta $(13-30 \mathrm{~Hz})$ frequency bands are crucial for various cognitive functions (e.g., attention control and episodic memory) and may help to explain the relationship between trait rumination and memory for contextual details. Our study uses EEG recorded during a source memory task to assess how alpha and beta oscillations during memory for contextual details may change as a function of trait rumination, anxiety, and depression level $(n=43)$. The source memory task instructs participants to remember objects and their associated contextual details. Memory for contextual details is lessened for participants higher in trait rumination paired with higher trait anxiety. Oscillations were analyzed in posterior parietal/occipital regions. During encoding, an interaction of nonclinical depression level and rumination predicts higher alpha power for items that were later not successfully remembered. During test, depression and rumination interact and predict higher alpha power for both successful and unsuccessful memory. These results suggest that trait anxiety, depression, and rumination impact accuracy and alpha oscillatory dynamics during contextual memory via changes in attention control.
\end{abstract}

Keywords Episodic memory $\cdot$ Cognitive flexibility $\cdot$ Rumination $\cdot$ Anxiety $\cdot$ Brain oscillations

Different patterns of neural activity observed as a function of trait rumination may provide information about how rumination might alter cognition. Rumination occurs when a person gets "stuck" on a thought or memory and cannot redirect attention away from the thought or memory (Nolen-Hoeksema, Wisco, \& Lyubomirsky, 2008). Clinical disorders, such as anxiety and depression, are linked to rumination, as well as difficulties with cognitive flexibility and memory functioning. For example, Liu et al. (2017) identified trait rumination as a partial mediator of the relationship between depression and episodic memory recall (e.g., memories based on episodes from our lives). Individuals with depression tend to

Nicole A. Forner-Phillips naf1016@wildcats.unh.edu

1 Department of Psychology and the Neuroscience and Behavior Program, The University of New Hampshire, 15 Academic Way, McConnell Hall Room 468, Durham, NH 03824, USA overgeneralize their episodic memories, leading to memory difficulties (Liu, Li, Xiao, Yang, \& Jiang, 2013). In particular, overgeneralized episodic memory leads to the person being unable to recall as many contextual details for an episode as they rely more on general schematic events (Soderlund et al. 2014). The observation that trait rumination is a partial mediator of the relationship between depression and episodic memory suggests that rumination itself plays a role in the decreased ability to remember specific contextual details from episodic memories. When a person has a high tendency to ruminate, they often get stuck on a thought and are unable to switch attention to a different thought, which could disrupt normal memory processes, leading to difficulties remembering specific contextual details. The power of brain oscillations in the alpha and beta oscillatory bands is negatively correlated with cognitive flexibility (Cooper et al., 2016; Cunillera et al., 2012; Jensen \& Mazaheri, 2010; Engel \& Fries, 2010; Foxe, Murphy, \& De Sanctis, 2014; Worden et al., 2000), and their examination may reveal how cognitive flexibility associated with rumination may impact episodic memory. 
Alpha oscillations $(8-12 \mathrm{~Hz})$ may be of importance for rumination, because they underlie several cognitive processes, including flexibility and memory. Task-switching tasks are associated with cognitive flexibility and show alpha power decreases in electrodes located over posterior parietal/ occipital areas in response to a switch cue but does not decrease in response to a cue indicating the current rule will be maintained (Buschman \& Miller, 2007; Buschman et al., 2012; Cooper et al., 2016; Cunillera et al., 2012; Foxe et al., 2014; Oswal et al., 2012; Phillips et al., 2014; Schmitz \& Voss, 2012; Thut et al., 2006; Wolff, Zink, Stock, \& Beste, 2017; Worden et al., 2000). The alpha power decrease during successful task-switching implies that decreases in alpha power in posteriorly located electrodes enable cognitive flexibility to occur, which also is in line with theories that propose alpha oscillations function to channel neural resources (Bonnefond, Kastner, \& Jensen, 2017; Hanslmayr, Staresina, \& Bowman, 2016; Jensen \& Mazaheri, 2010; Klimesch, 2012). Being stuck on a thought demonstrates an inability to flexibly shift attention away from a ruminative thought. According to the cognitive inflexibility hypothesis of rumination (Davis, \& Nolen-Hoeksema, 2000), this inability to flexibly shift attention is the result of impaired cognitive flexibility (Koster, De Lissnyder, Derakshan, \& De Raedt, 2011; Nejad, Fossati, \& Lemogne, 2013; Yee lo, Lau, Cheung, \& Allen, 2012), suggesting a possible link between alpha oscillatory function and rumination based on the demonstrated relationship between alpha power dynamics and cognitive flexibility. Importantly, behavioral data shows that people high in trait rumination (high ruminators) perform poorly on a task-switching task (Altamirano, Miyake, \& Whitmer, 2010) and on the Wisconsin card sorting task (Davis and Nolen-Hoeksema, 2000) compared with individuals low in trait rumination, signifying high trait rumination is associated with diminished cognitive flexibility. Difficulties with cognitive flexibility associated with higher trait rumination suggest that alpha power dynamics, which are associated with cognitive flexibility (Cooper et al., 2016; Cunillera et al., 2012; Foxe et al., 2014; Thut et al., 2006; Worden et al., 2000), may be disrupted for individuals high in trait rumination contributing to mental inflexibility and state rumination. Alpha power decreases in posteriorly located parietal/occipital electrodes associated with cognitive flexibility are also associated with successful memory encoding and retrieval (Clarke, Roberts, \& Ranganath, 2018; Düzel et al., 2003; Hanslmayr et al., 2009a, b, 2012, 2016; Khader \& Rösler, 2011; Klimesch et al., 1996, 1997; Mölle et al., 2002; Parish et al., 2018). This suggests that flexibility may be important for successful memory encoding and retrieval. Cognitive inflexibility associated with rumination could play a role in changes to episodic memory functioning in high ruminators. High ruminators may get stuck on a thought and be unable to flexibly shift attention to focus on encoding and/or retrieval of the details of an episode, which may lead to an inability to retrieve specific details. Rumination and alpha power dynamics have previously demonstrated relationships with cognitive flexibility; thus, observing how alpha oscillations relate directly with trait rumination may provide insight on whether cognitive inflexibility during episodic memory formation and retrieval in high ruminators impacts memory ability.

Alpha power plays a key role in episodic memory; it may help to bind item-in-context associations, possibly through attentionrelated mechanisms. Successful encoding and retrieval of a stimulus during recognition memory is linked to alpha power decreases in posterior brain regions (Clarke, Roberts, \& Ranganath, 2018; Düzel et al., 2003; Hanslmayr et al., 2009a, b, 2012, 2016; Khader \& Rösler, 2011; Klimesch et al., 1996, 1997; Mölle et al., 2002; Noh, Herzmann, Curran, \& De Sa, 2014; Parish et al., 2018). Importantly, Minarik, Berger, and Sauseng (2018) demonstrated that alpha power decreases in posterior parietal areas are important for the binding of an item and context during encoding and for the retrieval of contextual details during test. Although alpha power dynamics are important for successful encoding and retrieval of information, alpha power may not be reflective of a memory process per se. Alpha power during recognition memory is more likely reflective of differences in attentional demands (Klimesch, 2012; Van Driel et al., 2017). Therefore, alpha oscillation changes during recognition memory may be due to biasing of neural resources, which is a function of attention, towards areas that are active when encoding and retrieving a memory. Less alpha desynchronization during recognition memory may be reflective of fewer neural resources being devoted to the binding of item-context associations. If attention is split between a ruminative thought and a memory, then less neural resources may be available to bind an item with a context during encoding and/or to remember the item and context association during retrieval which may be reflected by less alpha desynchronization in posterior parietal/occipital brain regions.

Another factor that could play a part in memory for contextual details is the complexity of the neural code associated with a memory trace, which may be associated with beta (13$30 \mathrm{~Hz}$ ) oscillatory power. Hanslmayr et al. (2012) demonstrate that as beta oscillatory activity becomes increasingly desynchronized within a population of neurons, the potential complexity of firing patterns increases, which could increase the neural coding capacity allowing for more complex information to be stored and later retrieved. Thus, beta power decreases in posterior brain regions may enable the encoding and retrieval of stimulus specific features (Haegens, Vergara, Rossi-Pool, Lemus, \& Romo, 2017; Hanslmayr et al., 2012, 2014, 2016; Jafarpour, Fuentemilla, Horner, Penny, \& Duzel, 2014; Khader \& Rösler, 2011; Nyhus, 2018; Spitzer \& Haegens, 2017; Staudigl et al., 2015; Waldhauser, Braun, \& Hanslmayr, 2016). In practice, greater decreases in beta power in posterior brain regions during memory retrieval are linked to more detailed information being retrieved (Jafarpour et al., 
2014; Khader \& Rösler, 2011; Meconi et al., 2016; Nyhus, 2018; Waldhauser, Braun, \& Hanslmayr, 2016). For example, Waldhauser (2016) demonstrated that during successful recall of an item and a contextual detail, beta power decreased in the hemisphere contralateral to the side of the screen the object was initially presented on (the contextual detail). When the researchers applied TMS to one hemisphere in the beta frequency band during retrieval, memory for items encoded in the contralateral screen space was drastically impaired. The TMS prevented beta power from desynchronizing, which in turn led to impaired memory only for items that were encoded in the contralateral screen space. The fact that all memory was not impaired suggests that beta power dynamics may be related to specific features of a memory associated with a stimulus. Higher beta power in posterior brain regions associated with trait rumination could mean that less complex neural patterns could be expressed, which could lead to impoverished memory traces and decreased memory for contextual details.

Changes to alpha and beta power dynamics may underlie the relationship between trait rumination and episodic memory functioning. Putnam and McSweeney (2008) showed that clinically depressed individuals exhibit greater alpha power bilaterally in the prefrontal cortex while at rest, and the degree of increased power is associated with higher selfreported rumination. Ferdek, Van Rijn, and Wyczesany (2016) observed similar patterns in their study in which healthy young adults engaged in rumination of different valences (negative, neutral, or positive). Participants higher in trait rumination displayed elevated alpha power in the anterior cingulate cortex, increased low beta power within all brain regions examined (including posterior regions), and lower power in high beta in the dorsolateral prefrontal cortex for all conditions (Ferdek et al., 2016). These studies suggest that information carried in the beta band may be diminished, and alpha power may be elevated in individuals with a higher tendency to ruminate, both of which could negatively impact episodic memory formation and/or retrieval.

Higher alpha and beta power in posterior brain regions associated with trait rumination may underlie a diminished ability to remember contextual details, illustrating that changes to cognitive flexibility in individuals higher in trait rumination changes memory ability. Alpha and beta power dynamics may be disrupted in individuals high in trait rumination due to the cognitive inflexibility associated with rumination leading to weakened item-in-context associations. Based on the roles that alpha and beta oscillations play in cognitive flexibility and memory functioning, individuals higher in trait rumination may show a lessened ability to recall specific contextual details associated with a stimulus. Individuals higher in trait rumination are more likely to get stuck on a thought during encoding and/or retrieval. If an individual's neural resources are split between two different thoughts, it may hinder encoding and/or retrieval of stimulus specific features. To examine the hypothesis that altered alpha and beta power dynamics may underlie rumination's effect on episodic memory functioning, participants completed the Ruminative Responses Scale-Revised (RRS-R) to assess trait rumination followed by completion of a difficult source recognition memory task modeled after the task used in Ross \& Slotnick (2008) to assess memory for contextual details. Scores on the Beck Depression Inventory II and Beck Anxiety Inventory also were collected as control factors. Brain activity was recorded by EEG during the source memory task to assess any differences in alpha and beta power dynamics. Participants higher in trait rumination are more likely to get stuck on a thought during encoding and/or retrieval which could divert neural resources needed for a target memory and hinder the processing of more complex information leading to lessened memory ability. As such, we predicted that during encoding and retrieval, alpha and beta power decreases in posterior parietal/ occipital areas post stimulus presentation would be less drastic for participants with higher trait rumination, which would be associated with a decrease in their recall of contextual details associated with items.

\section{Materials and methods}

\section{Subjects}

Forty-seven participants were recruited from the University of New Hampshire Durham campus. Four participants were not included in the final analyses. Two participants were excluded for technical malfunctions of the EEG equipment and the other two participants were excluded, because their total item memory (old/hit/hit + old/hit/miss accuracy) was less than $50 \%$, indicating that participants were guessing during the test. Data from the remaining 43 participants (21 males) was used for analysis. The remaining participants had an average item $d$ prime of 0.75 , indicating that the participants were able to consistently differentiate the old and new stimuli and that they took the task seriously. Each participant gave written, informed consent to participate in accordance with the Institutional Review Board of the University of New Hampshire, which follows the guidelines of the Declaration of Helsinki. Each participant was between 18 and 35 years old (mean $=21.26$; standard deviation $[\mathrm{SD}]=3.92)$, had normal or corrected-to normal vision, and spoke English. Participants were ineligible if they had a learning disability, history of head trauma, serious cardiac issues, prior intracranial surgery, history or current neurological or psychiatric problems, or current use of antidepressants or anxiolytics. Participants were compensated with either an Amazon gift card (\$10 per hour) or course credit (1 credit per hour) for their participation in the study. The participants were allowed to choose which option they preferred for compensation. 


\section{Neuropsychological assessments}

The RRS-R was used to assess rumination as the revised version of the Ruminative Responses Scale has less depressive ideology making it a more accurate assessment of rumination (Treynor, Gonzalez, \& Nolen-Hoeksema, 2003). The RRS-R contains a series of statements regarding personal responses to feeling sad with a 4-point scale assessing how often the participant engages in that personal response. Participants also completed the Beck Depression Inventory-II (BDI-II; Beck, Steer, \& Brown, 1996) and the Beck Anxiety Inventory (BAI; Beck, Epstein, Brown, \& Steer, 1988). Scores from the BDI-II and the BAI were collected as control factors for the analyses.

Participants also were administered a battery of neuropsychological assessments to assess intelligence, working memory capacity, and executive functioning. The Weschler Test of Adult Reading (WTAR; Pearson, 2001) was used to assess general intelligence, the forward and backward digit span (Weschler, 1981) was used to assess working memory capacity, and the Trail Making Test parts A \& B (Army individual test battery, 1944) were used to assess processing speed and executive function.

\section{Source memory task}

The source memory task was presented using E-prime 2.0 (Psychology Software Tools Inc, Sharpsburg, PA) and used 192 different non-nameable objects (originally created for Slotnick and Schacter, 2004) as stimuli across 6 study-test runs. A study-test run consisted of a study phase of 32 items followed immediately by a test phase of 48 items, the 32 old stimuli plus 16 new stimuli (Fig. 1). The non-nameable objects were constructed by generating pseudorandom Bezier curves, and then the objects were filled with lines with different colors and orientations. Non-nameable objects were used to make the task more difficult, to ensure a range of performance was obtained, and to increase the chances rumination occurred during the task. The objects used were counterbalanced across source conditions (left and right side of screen) and the six study-test runs were counterbalanced across participants. The task involved one practice study-test run followed by six experimental study-test runs.

During each experimental study phase, the participants were presented with 16 objects on the left side of the screen and 16 objects on the right side of the screen for a total of 32 objects. The order of the presentation was pseudo-random so that no more than 3 objects in a row were presented on the same side. During study, each object was presented on screen for 2.5 seconds, and there was a 1.5 second break between objects. A fixation cross remained on screen during study to use as a reference point for which side of the screen the object was on. After the study phase, the participants received an eight second break before starting the test phase. During the experimental test phase, all objects were presented in the center of the screen. Sixteen new objects were mixed in with the 32 previously studied objects for a total of 48 objects presented during the test phase. Stimuli during test were presented in a pseudorandom order where no more than three of the same condition in a row could occur. For each object presented during test, participants were instructed to press " 1 " if they remembered the object was previously studied on the left side of the screen, " 2 " if they believed the object was not previously studied, or " 3 " if they remembered the object was previously studied on the right side of the screen. Each object presented during the test phase was on screen for 2.5 seconds followed by a fixation cross. The fixation cross duration was an average of three seconds with a range from 2 seconds to 4 seconds in 200-millisecond increments. During the experimental trials, E-prime collected data on accuracy and reaction time. When participants correctly recalled an item along with its contextual detail, their response was termed an old/hit/hit. When participants correctly recalled the item, but not the

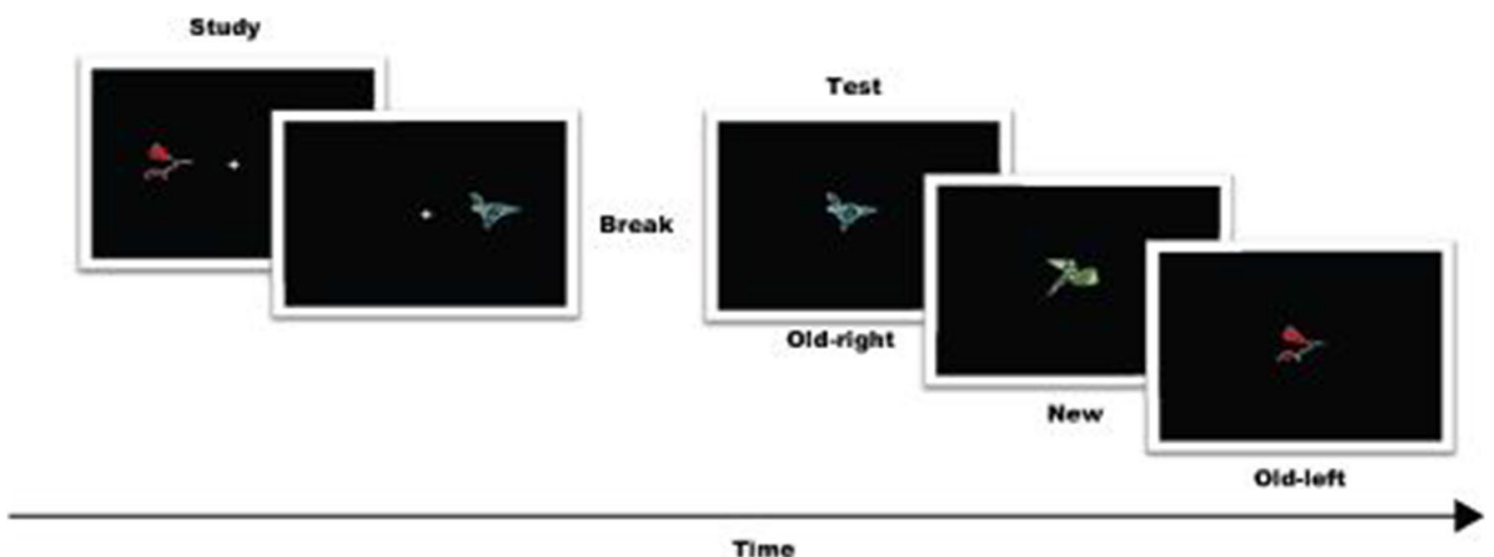

Time

Fig. 1 Source memory task procedure. During study, items are presented on the left side of the screen or the right side of the screen. After study, there is a brief break. During the test, all items are presented in the center of the screen. Each item is separated by a fixation cross of variable duration. During the test, the participant denoted their memory decision with a button press. Old-right is an object that was previously studied on the right side of the screen. New is an object not seen during study. Oldleft is an object that was previously studied on the left side of the screen 
contextual detail, their response was termed an old/hit/miss. If a participant responded that an item was new, but it was one of the objects presented during study, the response was termed a miss.

Before EEG was recorded and E-prime started collecting data, participants were given a practice version of the source memory task. The practice version used the same study-test procedure as the experimental version with fewer stimuli. The practice version had a total of 20 non-nameable objects during the study phase, 10 presented on the left and 10 presented on the right side of the screen. During test, 10 new objects were mixed in with the objects from the study phase for a total of 30 objects during test. After the practice study-test trial was complete, the researcher asked participants if they had any questions before moving on to the experimental study-test trials to ensure that the participants understood the goal of the task.

\section{Behavioral analysis}

An alpha level of 0.05 was used for all behavioral comparisons. Proportion of correct response (accuracy) values were extracted from E-prime and analyzed in SPSS v25 (IBM Corporation, Armonk, NY). Behavioral performance was assessed by performing two linear regression analyses. The dependent variables for the two different linear regressions were old/hit/hit proportion and old/hit/miss proportion. RRS$\mathrm{R}, \mathrm{BDI}-\mathrm{II}$, and BAI scores were used as predictor variables for each analysis along with their interaction terms. Centered scores (individual score-mean score) were used to create the interaction terms. All predictor variables were entered in to the linear regression model at the same time (forced entry).

\section{EEG data acquisition}

EEG data were recorded in a radiofrequency-shielded room (Universal Shielding Corp., Deer Park, NY) from a 64channel BrainVision ActiChamp EEG system with $\mathrm{Ag}-\mathrm{AgCl}$ electrodes (Brain Products GmbH, Munich, Germany). Data were collected using BrainVision's Pycorder software, vertex referenced, and sampled at $500 \mathrm{~Hz}$ using an analog filter of 0.1-200 Hz. Electrode impedances were kept below $25 \mathrm{k} \Omega$. The P1 electrode could not be kept below $25 \mathrm{k} \Omega$ for four participants, so the channel was excluded from the final EEG analysis for those four participants.

\section{EEG data analysis}

EEG data was processed using the MATLAB (Mathworks, Natick, MA) plug-in EEGLAB (Delorme \& Makeig, 2004). The EEGLAB tool for automatic channel rejection was used to identify any channels with kurtosis greater than five standard deviations from the mean for that channel. Any channels identified by the automatic channel rejection tool were not removed from analysis, but they were excluded only when the EEG data were re-referenced. Before re-referencing, the data were filtered between 1 and $100 \mathrm{~Hz}$. After the data were filtered, it was re-referenced to the average EEG signal excluding any artifact laden channels identified by the automatic channel rejection tool. An average of three channels $(\mathrm{SD}=2)$ were excluded from rereferencing for each participant. The data, including the identified artifact laden channels, was then sorted into epochs from one second prior to two seconds after stimulus onset. The resulting epochs were visually inspected for any nonblink-related changes in voltage greater than 75 microvolts, which were removed from further analysis. Once epoch rejection was complete, Infomax Independent Component Analysis (ICA) (Bell \& Sejnowski, 1995) was used to separate the data into independent components. Epochs for the resulting independent components were visually inspected and noise-related epochs were excluded from further analysis. Noise-related epochs were identified by uniform large changes in voltage. After noise-related epochs were removed, Infomax ICA was run again. An average of 144 epochs $(\mathrm{SD}=71)$ were rejected for each participant. The EEGLAB plug-in function ADJUST (Mognon et al., 2011) was used to identify artifact components for each participant. Identified artifact components were excluded from further analysis, and data were converted back into sensor space. All oscillatory analyses were done using channel data in sensor space.

Electrodes P5, P3, P1, Pz, P2, P4, P6, PO3, POz, and PO4 (Fig. 2) were selected a priori for analysis based on previous studies examining alpha and beta power during recognition memory (Clarke, Roberts, \& Ranganath, 2018; Düzel et al., 2003; Hanslmayr et al., 2009a, b, 2012, 2016; Khader \& Rösler, 2011; Klimesch et al., 1996; Waldhauser, Braun, \& Hanslmayr, 2016). Event-related spectral perturbation maps (ERSPs), which specify stimulus evoked power, were made using single trial data from each channel for old/hit/hits, old/ hit/misses, and miss trials. Miss trial activity was combined with old/hit/miss trial activity to increase statistical power, because many participants had less than 30 trials for old/hit/ miss activity alone. We felt justified in combining old/hit/miss and miss responses, because there is no memory for the contextual detail in either condition. The average final number of old/hit/hit trials per participant was $66(\mathrm{SD}=26.15)$, and the average final number of trials for old/hit/miss combined with miss trials per participant was $64(\mathrm{SD}=19.38)$. Timefrequency decomposition was performed using a modified Morlet wavelet in 25-ms steps for $30 \mathrm{log}$-spaced frequencies between 4 and $50 \mathrm{~Hz}$ with 2 cycles at $4 \mathrm{~Hz}$ and 5 cycles at 50 Hz. Epochs were trimmed at each edge to avoid boundary effects. ERSP values of interest were averaged across all 10 electrodes of interest and were then averaged across frequency. Alpha power was averaged between $8-13 \mathrm{~Hz}$, whereas beta power was averaged between $14-25 \mathrm{~Hz}$. 


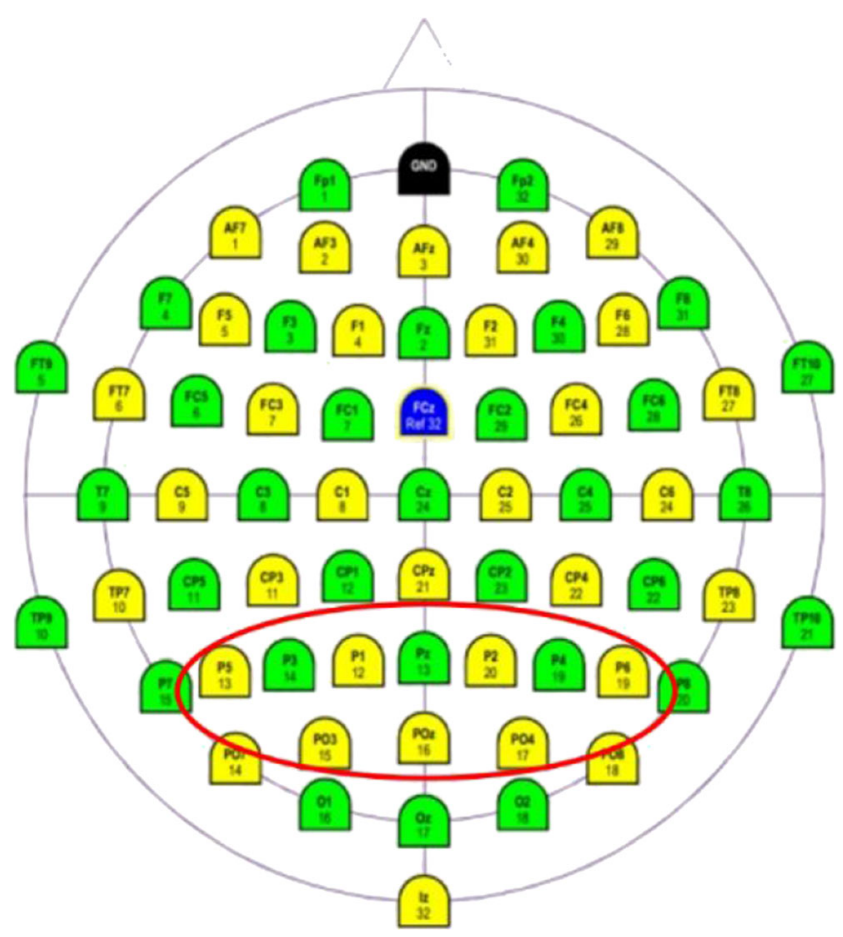

Fig. 2 Electrodes used in EEG analysis. Pictured is a diagram of the layout of electrodes for the 64 channel Brain Vision actichamp EEG system. The triangle at the top of the image represents a nose in order to orient where on the scalp the electrodes are. The red oval highlights the electrodes used for EEG analysis in our study. The electrodes used cover mostly parietal brain areas

Trial-by-trial mean alpha and beta power values were extracted from EEGLAB to be further analyzed in $\mathrm{R}$ ( $\mathrm{R}$ core team, Vienna, Austria). After extraction, the power values for each trial were baseline-corrected by subtracting the total trial mean power value from each individual power value within the trial and dividing the resulting number by the standard deviation of the trial (Grandchamp \& Delorme, 2011). The power values for each trial were then averaged into six different time bins between 0 and 1,500 milliseconds post cue in order to better capture the variability within the EEG data. The final power values reflect the average percent change in power from baseline.

We sought to test whether tendency to ruminate, depression, or anxiety predict mean percent change in alpha and beta power from baseline during both encoding and retrieval of stimuli within trial type (old/hit/hit, old/hit/miss+miss). We explored this question by constructing a series of mixed effects linear regressions. Mixed effects linear regressions are ideal for this type of repeated measures data because there are multiple observations (e.g., trials) per person. Participant was included as a random effect in all models to account for baseline differences across participants (Baayen, Davidson, \& Bates, 2008; Murphy, \& Dunne, 2005). A total of eight mixed effects linear regressions were constructed (4 for encoding; 4 for retrieval). A linear mixed effects model was constructed for each clinical symptomatology questionnaire separately (BDI-II, and BAI scores) with rumination score (RRS-R). The models were constructed in this way to attempt to limit the number of predictor variables in each regression but also account for any interactions of anxiety and depression with rumination. Change in alpha/beta power was regressed on scores from the questionnaires (continuous fixed effect), trial type (old/hit/hit or old/hit/miss+miss), and their interaction terms. Interaction terms between rumination/depression and rumination/anxiety were also included to test whether the subclinical measures moderated the effect of trial type on percent change in alpha or beta power. Because multiple comparisons were used, the typical alpha level of 0.05 was divided by the number of comparisons ( 8 regressions) per the Bonferroni method to create a corrected $p$ value of 0.006 , which was used to determine significance. A significant interaction of any of the clinical measures with trial type prompted follow-up regressions to determine in which direction the effects were.

Follow-up mixed effects linear regressions were used to further explore significant interaction terms. Separate nested regressions were run for each trial type separately (old/hit/hit and old/hit/miss+miss) to test how clinical measures were related to change in alpha/beta under separate trial type conditions. The Bonferroni corrected $p$ value of 0.006 was used to determine significance for all follow-up regressions.

\section{Results}

\section{Behavioral results}

Rumination, depression, anxiety, and their interaction terms (rumination $\mathrm{x}$ depression, rumination $\mathrm{x}$ anxiety, and depression $\mathrm{x}$ anxiety) were used as predictor variables in standard linear regressions with proportion of responses for old/hit/hits and old/hit/misses as dependent variables. For significant independent predictor variables, the part correlation squared $\left(s r^{2}\right), b$, standard error of $b(S E b)$, and standardized beta value are reported. The part correlation squared represents the unique proportion of variance that can be explained by an individual predictor variable when controlling for the other predictor variables in the model. Means and standard deviations for each variable can be found in Table 1. None of the predictor variables or dependent variables had outliers, impossible scores, or missing scores. All quantitative variables were normally distributed, and pairs of quantitative variables had a normal bivariate distribution, no evidence of heteroscedasticity, and there were no bivariate outliers. An alpha level of 0.05 was used for all behavioral comparisons.

A standard linear regression was used to examine whether rumination, depression, anxiety, and their interactions predict memory for contextual details (old/hit/hit proportion correct). Zero order correlations between variables can be found in 
Table 1. Means and standard deviations of variables

\begin{tabular}{lll}
\hline Variables & Mean & Standard deviation \\
\hline RRS-R & 20.21 & 3.99 \\
BDI-II & 5.53 & 4.31 \\
BAI & 5.51 & 5.43 \\
OHH proportion & 0.51 & 0.11 \\
OHM proportion & 0.25 & 0.07 \\
OHH reaction time (seconds) & 1.73 & 0.61 \\
OHM reaction time (seconds) & 1.86 & 0.62 \\
Average \% Change Alpha Encoding OHH & 0.51 & 0.75 \\
Average \% Change Alpha Encoding OHM/M & 0.44 & 0.77 \\
Average \% Change Beta Encoding OHH & 0.24 & 0.61 \\
Average \% Change Beta Encoding OHM/M & 0.19 & 0.60 \\
Average \% Change Alpha Retrieval OHH & 0.39 & 0.72 \\
Average \% Change Alpha Retrieval OHM/M & 0.34 & 0.73 \\
Average \% Change Beta Retrieval OHH & 0.11 & 0.56 \\
Average \% Change Beta Retrieval OHM/M & 0.07 & 0.56 \\
\hline
\end{tabular}

Notes: Ruminative Responses Scale-Revised (RRS-R), Beck Depression Inventory-II (BDI-II), Beck Anxiety Inventory (BAI), Old/hit/hit (OHH), Old/ hit/miss $(\mathrm{OHM}), \mathrm{Old} / \mathrm{hit} / \mathrm{miss}+\mathrm{miss}(\mathrm{OHM} / \mathrm{M})$. Average \% change reflects the average \% change in power from baseline. Baseline is the average power post cue for each trial

Table 2 . The overall regression model was significant $(R=$ $0.53, F(6,36)=2.39, p=0.048)$. Combined, rumination, depression, anxiety, and their interactions predict $28.5 \%$ of the variance in old/hit/hit proportion. Importantly, the only predictor variable that could uniquely predict a significant proportion of variance in old/hit/hit proportion when controlling for all other variables was the interaction of rumination and anxiety $\left(s r^{2}=0.210, b=-0.005\right.$, $S E b=0.001$, standardized beta $=-1.051 ; t(36)=-3.25, p$ $=0.003$; Fig. 3A; Table 3). The information presented in Fig. 3, and all other figures that depict a significant interaction of predictor variables, uses values we selected to illustrate the regression equation. For example, we chose to graphically represent what the regression equation looks like for both the highest and lowest rumination value obtained in our data set at different levels of trait anxiety. The relationship between rumination and old/hit/hit proportion appears to become more negative with increasing anxiety. When rumination and anxiety were higher, participants had fewer trials in which they correctly remembered both the item and the contextual detail associated with the item.

A standard linear regression was also used to examine whether rumination, depression, anxiety, and their

Table 2. Zero-order correlations of variables for behavior regressions

\begin{tabular}{|c|c|c|c|c|c|c|c|c|c|c|}
\hline & $\begin{array}{l}\mathrm{OHH} \\
\text { proportion }\end{array}$ & $\begin{array}{l}\text { OHM } \\
\text { proportion }\end{array}$ & $\begin{array}{l}\mathrm{OHH} \\
\mathrm{RT}\end{array}$ & $\begin{array}{l}\text { OHM } \\
\text { RT }\end{array}$ & $\begin{array}{l}\text { RRS- } \\
\mathrm{R}\end{array}$ & $\begin{array}{l}\text { BDI- } \\
\text { II }\end{array}$ & BAI & $\begin{array}{l}\text { Interaction of } \\
\text { RRS-R and } \\
\text { BDI-II }\end{array}$ & $\begin{array}{l}\text { Interaction of RRS-R } \\
\text { and BAI }\end{array}$ & $\begin{array}{l}\text { Interaction of BDI-II } \\
\text { and BAI }\end{array}$ \\
\hline OHH proportion & 1 & - & - & - & 0.18 & 0.10 & 0.07 & -0.12 & -0.28 & -0.03 \\
\hline OHM proportion & - & 1 & - & - & 0.12 & 0.18 & 0.25 & 0.26 & 0.53 & 0.32 \\
\hline OHH RT & - & - & 1 & - & -0.28 & -0.14 & -0.19 & -0.21 & -0.28 & -0.13 \\
\hline OHM RT & - & - & - & 1 & -0.28 & -0.15 & -0.21 & -0.26 & -0.33 & -0.17 \\
\hline RRS-R & 0.18 & 0.12 & -0.28 & -0.28 & 1 & 0.37 & 0.39 & 0.23 & .20 & 0.24 \\
\hline BDI-II & 0.10 & 0.18 & -0.14 & -0.15 & 0.37 & 1 & 0.78 & 0.48 & .48 & 0.69 \\
\hline BAI & 0.07 & 0.25 & -0.19 & -0.21 & 0.39 & 0.78 & 1 & 0.50 & 0.66 & 0.80 \\
\hline $\begin{array}{l}\text { Interaction of RRS-R } \\
\text { and BDI-II }\end{array}$ & -0.12 & 0.26 & -0.21 & -0.26 & 0.23 & 0.48 & 0.50 & 1 & 0.80 & 0.71 \\
\hline $\begin{array}{l}\text { Interaction of RRS-R } \\
\text { and BAI }\end{array}$ & -0.28 & 0.53 & -0.28 & -0.33 & 0.20 & 0.48 & 0.66 & 0.80 & 1 & 0.83 \\
\hline $\begin{array}{l}\text { Interaction of BDI-II } \\
\text { and BAI }\end{array}$ & -0.03 & 0.32 & -0.13 & -0.17 & 0.24 & 0.69 & 0.80 & 0.71 & 0.83 & 1 \\
\hline
\end{tabular}

Notes: Ruminative Responses Scale-Revised (RRS-R), Beck Depression Inventory-II (BDI-II), Beck Anxiety Inventory (BAI), Old/hit/hit (OHH), Old/ $\mathrm{hit} / \mathrm{miss}(\mathrm{OHM}), \mathrm{Old} / \mathrm{hit} / \mathrm{miss}+\mathrm{miss}(\mathrm{OHM} / \mathrm{M})$ 
a

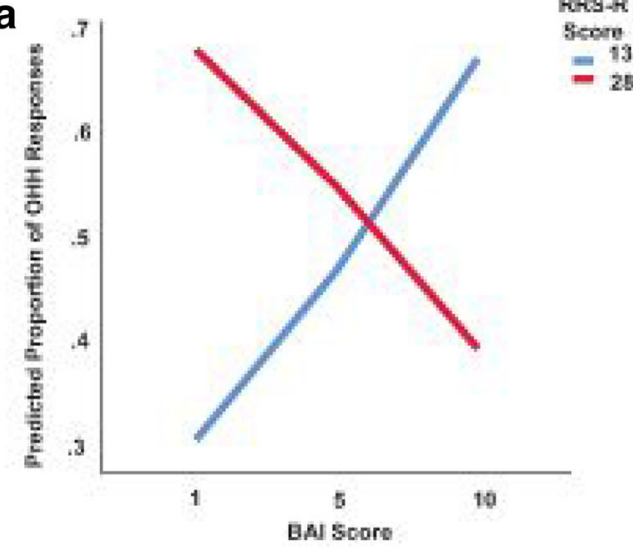

b

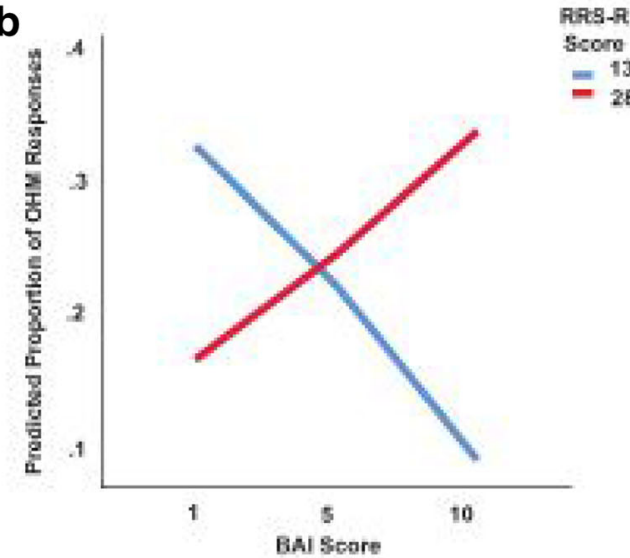

C

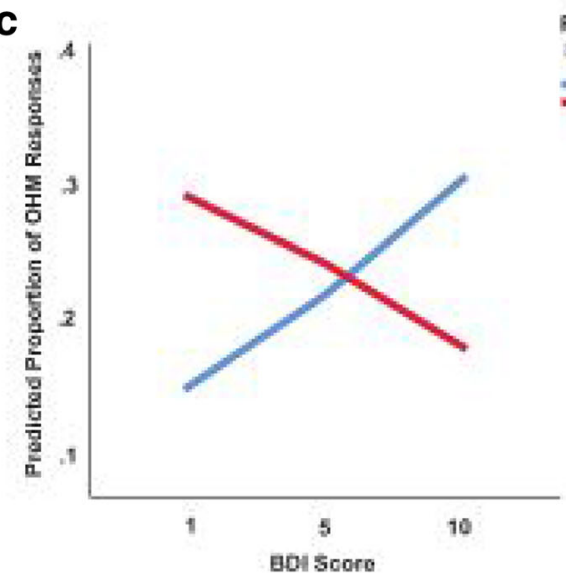

Fig. 3 Graphical representation of the regression equations for behavioral analysis. The graphs display predicted proportion of responses for old/hit/ hits $(\mathrm{OHH})$ and old/hit/misses $(\mathrm{OHM})$ based on interactions between the predictor variables. A range of scores for each variable were chosen and entered into the regression equation to produce the points depicted. The scores chosen for the variables on the $\mathrm{x}$-axis are the mean for the predictor variable and the scores that are one standard deviation on either side of the mean. The scores chosen for rumination were the highest (26; red line) and lowest (13; blue line) RRS-R score from our sample. The score chosen for the variable not represented in the interaction was the mean for that score. (A) The interaction of anxiety and rumination predicting proportion of old/hit/hit responses. (B) The interaction of anxiety and rumination predicting the proportion of old/hit/miss responses. (C) The interaction of depression and rumination predicting the proportion of old/hit/miss responses interactions predict memory for the target item without the contextual detail (old/hit/miss proportion). Zero order correlations between variables can be found in Table 2 . The overall regression model was significant $(R=0.64$, $F(6,36)=4.17, p=0.003)$. Combined, rumination, depression, anxiety, and their interactions predict $41.0 \%$ of the variance in old/hit/miss proportion. As with old-hit-hit proportion, the interaction of rumination and anxiety uniquely predicts a significant proportion of variance in old/hit/miss proportion when controlling for all other variables $\left(s r^{2}=0.30, b=0.003, S E b=0.001\right.$, standardized beta $=1.254 ; t(36)=4.27, p<0.001$; Fig. 3B; Table 4). The relationship between rumination and old/hit/miss proportion appears to become more positive with increasing anxiety. When rumination and anxiety are higher, participants made more judgements in which they correctly remembered the item but did not remember the contextual detail. The interaction of rumination and depression also could uniquely predict a significant proportion of variance in old/hit/miss proportion when controlling for all other variables $\left(s r^{2}=0.086, b=-0.002, S E b=0.001\right.$, standardized beta $=-0.53 ; t(36)=-2.30, p=0.027$; Fig. 3C; Table 4). The relationship between rumination and old/hit/miss proportion appears to become more negative with increasing depression. When rumination and depression are higher, participants made less judgements in which they correctly remembered the item but did not remember the contextual detail.

\section{ERSP results}

To answer the question of whether tendency to ruminate could account for a significant proportion of variance in mean percent change in alpha and beta power per trial type while also accounting for anxiety and depression, a trial-by-trial analysis with separate mixed effects regressions were used with rumination interacting with anxiety and rumination interacting

Table 3. Results from $\mathrm{OHH}$ proportion regression

\begin{tabular}{llll}
\hline & $\mathrm{b}$ & Standardized beta & $\mathrm{Sr}^{2}$ \\
\hline RRS-R & 0.004 & 0.147 & 0.017 \\
BDI-II & -0.003 & -0.115 & 0.004 \\
BAI & 0.006 & 0.272 & 0.016 \\
Interaction of RRS-R and BDI-II & 0.002 & 0.266 & 0.022 \\
Interaction of RRS-R and BAI & $-0.005^{*}$ & $-1.051^{*}$ & $0.210^{*}$ \\
Interaction of BDI-II and BAI & 0.001 & 0.485 & 0.041 \\
\hline
\end{tabular}

Notes: Asterisk denotes significance at the 0.05 level. Ruminative Responses Scale-Revised (RRS-R), Beck Depression Inventory-II (BDI-II), Beck Anxiety Inventory (BAI), Old/hit/hit (OHH), Old/hit/miss $(\mathrm{OHM}), \mathrm{Old} / \mathrm{hit} / \mathrm{miss}(\mathrm{OHM}), \mathrm{b}=$ beta, and $\mathrm{Sr}^{2}$ is the unique variance explained by a predictor variable 
Table 4. Results from OHM proportion regression

\begin{tabular}{llll}
\hline & $\mathrm{B}$ & Standardized beta & $\mathrm{Sr}^{2}$ \\
\hline RRS-R & 0.002 & 0.093 & 0.007 \\
BDI-II & 0.003 & 0.207 & 0.014 \\
BAI & -0.004 & -0.300 & 0.020 \\
Interaction of RRS-R and BDI-II & $-0.002 *$ & $-0.53^{*}$ & $0.086^{*}$ \\
Interaction of RRS-R and BAI & $0.003 *$ & $1.254^{*}$ & $0.300^{*}$ \\
Interaction of BDI-II and BAI & 0.000 & -0.27 & 0.013 \\
\hline
\end{tabular}

Notes: Asterisk denotes significance at the 0.05 level. Ruminative Responses Scale-Revised (RRS-R), Beck Depression Inventory-II (BDI-II), Beck Anxiety Inventory (BAI), Old/hit/hit (OHH), Old/hit/miss $(\mathrm{OHM}), \mathrm{Old} / \mathrm{hit} / \mathrm{miss}(\mathrm{OHM}), \mathrm{b}=$ beta, and $\mathrm{Sr}^{2}$ is the unique variance explained by a predictor variable

with depression as predictor variables. For each main regression, an interaction of any of the predictor variables with trial type prompted further dissociation. The main analysis resulted in eight mixed-effects linear regressions (4 for encoding and 4 for retrieval) with a Bonferroni corrected $p$ value of 0.006 used to determine significance.

Significant interactions with trial type were found for depression during encoding predicting alpha (degrees of free$\operatorname{dom}[d f]=1, \mathrm{~b}=-0.03, \mathrm{X}^{2}=12.49, p<0.001$; Table 5), the interaction of depression and rumination during encoding predicting alpha $\left(d f=1, b=0.001, \mathrm{X}^{2}=13.84, p<0.001\right.$; Table 5), anxiety during encoding predicting beta $(d f=1, b=$ $-0.01, \mathrm{X}^{2}=8.20, p=0.004$; Table 6), depression during retrieval predicting alpha $\left(d f=1, b=-0.02, \mathrm{X}^{2}=18.48, p<\right.$ 0.001 ; Table 5), the interaction of rumination and depression during retrieval predicting alpha $\left(d f=1, b=0.001, \mathrm{X}^{2}=8.51\right.$, $p=0.004$; Table 5), and anxiety during retrieval predicting alpha $\left(d f=1, b=-0.001, \mathrm{X}^{2}=41.67, p<0.001\right.$; Table 5). A summary of results from all eight regressions can be found in Tables 5 and 6. Each significant interaction with trial type was further explored by separating the trial types in to old/hit/hit and old/hit/miss+miss and running follow-up linear regressions to see in which direction the effect was within each trial type, resulting in 12 follow-up regressions. The degrees of freedom on the follow up regressions reflect the number of data points analyzed for each trial type. Because power values were separated into six time bins post cue per trial, each time bin counts as a data point in the model, leading to a large number of data points to help capture variability within the data. A summary of results from each follow-up regression can be found in Table 11.

\section{Alpha results}

Encoding. When the finding of depression and rumination predicting mean alpha power change during encoding was further analyzed, we found that during a trial in which an item was encoded that was later an old/hit/hit, for each one unit increase in BDI-II score, alpha power decreased by $0.002 \%$ $(F(1,16274)=108.21, p<0.001$; Fig. 4A) and for each one unit increase in RRS-R score, alpha power increased by $0.008 \%(F(1,16274)=9.34, p=0.002$; Fig. 4B). During a trial in which an item was encoded that was later an old/hit/ miss or miss, for each one unit increase in BDI-II score, alpha power decreased by $0.048 \%(F(1,15746)=16.46, p<0.001)$, and there was a significant interaction between depression and rumination $(F(1,15746)=25.74, p<0.001$; Fig. $4 C)$. The relationship between depression and mean percent change in alpha power became more positive with increasing rumination.

Retrieval. When depression and rumination predicting mean alpha power change during retrieval was further analyzed, we found that during an old/hit/hit trial there was a significant interaction of depression and rumination $(F(1,17996)=17.11, p<0.001$; Fig. 5A). The relationship between depression and mean percent change in alpha power became more positive with increasing rumination. During an $\mathrm{old} / \mathrm{hit} / \mathrm{miss}$ or miss trial, for each one unit increase in BDI-II score, alpha power decreased by $0.029 \%(F(1,17216)=$ $111.51, p<0.001)$, for each one unit increase in RRS-R score, alpha power decreased by $0.024 \%(F(1,17216)=83.14, p<$ $0.001)$, and there was a significant interaction of depression and rumination $(F(1,17216)=35.40, p<0.001$; Fig. 5B). When anxiety predicting mean alpha power change during retrieval was further analyzed, we found that during an old/ hit/hit trial for each one unit increase in BAI score, alpha power decreased by $0.003 \%(F(1,17998)=7.73, p=0.005$; Fig. 5C). During an old/hit/miss or miss trial, for each one unit increase in BAI score, alpha power increased by $0.009 \%$ $(F(1,17218)=91.10, p<0.001 ;$ Fig. 5D).

\section{Beta results}

When anxiety predicting mean beta power change during encoding was further analyzed, we found that during a trial in which an item was encoded that was later an old/hit/hit, for each one unit increase in BAI score, beta power decreased by $0.008 \%(F(1,16276)=74.24, p<0.001$; Fig. 6A $)$. During a trial in which an item was encoded that was later an old/hit/ miss or miss, for each one unit increase in BAI score, beta power decreased by $0.003 \%(F(1,15748)=10.23, p=0.001$; Fig. 6B).

\section{Discussion}

Trait rumination, anxiety, and depression may decrease memory performance through changes in cognitive flexibility and the complexity of a memory trace during memory for contextual details. Our results show that trait rumination, anxiety, 
Table 5. Results from mixed effects linear regressions with alpha power change as the dependent variable

\begin{tabular}{|c|c|c|c|c|}
\hline & Variable & $\mathrm{B}$ & $X^{2}$ & $P$ \\
\hline \multirow[t]{7}{*}{ Encoding Alpha RRS-R and BAI } & RRS-R & 0.471 & 0.015 & 0.903 \\
\hline & BAI & 0.005 & 0.641 & 0.423 \\
\hline & Trial Type & $0.114 *$ & $35.34 *$ & $<0.001 *$ \\
\hline & RRS-R by BAI & -0.001 & 0.507 & 0.477 \\
\hline & RRS-R by trial type & -0.009 & 1.247 & 0.264 \\
\hline & BAI by trial type & -0.025 & 5.921 & 0.008 \\
\hline & RRS-R by BAI by trial type & 0.001 & 7.072 & 0.008 \\
\hline \multirow[t]{7}{*}{ Encoding Alpha RRS-R and BDI-II } & RRS-R & 0.017 & 0.000 & 0.986 \\
\hline & BDI-II & 0.027 & 1.264 & 0.261 \\
\hline & Trial type & $0.196^{*}$ & $35.249 *$ & $<0.001 *$ \\
\hline & RRS-R by BDI-II & -0.002 & 0.133 & 0.716 \\
\hline & RRS-R by trial type & -0.014 & 2.417 & 0.120 \\
\hline & BDI-II by trial type & $-0.034 *$ & $12.492 *$ & $<0.001 *$ \\
\hline & RRS-R by BDI-II by trial type & $0.002 *$ & $13.837 *$ & $<0.001 *$ \\
\hline \multirow[t]{7}{*}{ Retrieval Alpha RRS-R and BAI } & RRS-R & -0.002 & 0.700 & 0.403 \\
\hline & BAI & -0.013 & 0.478 & 0.489 \\
\hline & Trial type & 0.011 & 6.24 & 0.012 \\
\hline & RRS-R by BAI & 0.000 & 0.596 & 0.440 \\
\hline & RRS-R by trial type & -0.009 & 4.092 & 0.043 \\
\hline & BAI by trial type & $-0.011 *$ & $41.667 *$ & $<0.001 *$ \\
\hline & RRS-R by BAI by trial type & 0.001 & 4.755 & 0.029 \\
\hline \multirow[t]{7}{*}{ Retrieval Alpha RRS-R and BDI-II } & RRS-R & -0.003 & 0.729 & 0.393 \\
\hline & BDI-II & 0.003 & 0.662 & 0.416 \\
\hline & Trial type & 0.128 & 6.202 & 0.013 \\
\hline & RRS-R by BDI-II & -0.000 & 0.574 & 0.449 \\
\hline & RRS-R by trial type & -0.009 & 1.056 & 0.304 \\
\hline & BDI-II by trial type & $-0.021 *$ & $18.478^{*}$ & $<0.001 *$ \\
\hline & RRS-R by BDI-II by trial type & $0.001 *$ & $8.506^{*}$ & $0.004 *$ \\
\hline
\end{tabular}

Notes: Asterisk denotes significance at the 0.006 level. Ruminative Responses Scale-Revised (RRS-R), Beck Depression Inventory-II (BDI-II), Beck Anxiety Inventory (BAI), Old/hit/hit (OHH), Old/hit/miss (OHM), Old/hit/miss (OHM), b = beta

and depression are all associated with altered alpha and beta oscillatory power dynamics during memory for contextual details. Specifically, the ability to remember contextual details is decreased in people who have higher trait anxiety levels paired with higher trait rumination. Linear regression analyses of proportion of responses for old/hit/hits and old/hit/misses revealed that as trait rumination increases, old/hit/hit proportion decreases (Fig. 3A), and old/hit/miss proportion increases (Fig. 3B), but only with higher trait anxiety. Also, higher trait rumination paired with higher levels of nonclinical depression leads to fewer old/hit/miss trials (Fig. 3C). Our original hypothesis was that trait rumination would be able to predict memory for contextual details on its own. However, rumination is often a symptom of depression and anxiety, so it is not surprising that rumination has interactions with both depression and anxiety levels. Additionally, the interactions of trait rumination with trait anxiety are in the predicted direction.
Our trial-by-trial analysis reveals several changes in alpha and beta oscillatory activity in electrodes located over posterior parietal/occipital areas, which may be related to the changes in memory. Higher levels of nonclinical depression are associated with greater power decreases for alpha during encoding for trials in which an item was later an old/hit/hit (Fig. 4A). For items that were later old/hit/misses or misses, the relationship between depression and alpha power becomes more positive with increasing trait rumination (Fig. 4C). Also, as trait rumination increases, alpha power during encoding for items that are later old/hit/hits increases (Fig. 4B). Higher levels of trait anxiety during encoding predict greater power decreases in beta power for both items that are later old/hit/hits (Fig. 6A) and items that are later old/hit/miss or misses (Fig. $6 \mathrm{~B})$. During retrieval, higher trait anxiety is associated with decreased alpha power for old/hit/hits (Fig. 5C) and increased alpha power during an old/hit/miss or miss trial (Fig. 5D). 
Table 6. Results from mixed effects linear regressions with beta power change as the dependent variable

\begin{tabular}{|c|c|c|c|c|}
\hline & Variable & $\mathrm{b}$ & $X^{2}$ & $P$ \\
\hline \multirow[t]{7}{*}{ Encoding Beta RRS-R and BAI } & RRS-R & 0.100 & 0.686 & 0.408 \\
\hline & BAI & 0.004 & 3.066 & 0.080 \\
\hline & Trial type & $0.071 *$ & $29.503 *$ & $<0.001 *$ \\
\hline & RRS-R by BAI & -0.001 & 0.084 & 0.772 \\
\hline & RRS-R by trial type & -0.006 & 2.834 & 0.092 \\
\hline & BAI by trial type & $-0.011 *$ & $8.204 *$ & $0.004 *$ \\
\hline & RRS-R by BAI by trial type & 0.001 & 2.816 & 0.093 \\
\hline \multirow[t]{7}{*}{ Encoding Beta RRS-R and BDI-II } & RRS-R & 0.008 & 1.151 & 0.283 \\
\hline & BDI-II & -0.013 & 5.920 & 0.015 \\
\hline & Trial type & $0.034^{*}$ & $29.521^{*}$ & $<0.001 *$ \\
\hline & RRS-R by BDI-II & -0.000 & 0.200 & 0.656 \\
\hline & RRS-R by trial type & -0.005 & 2.323 & 0.127 \\
\hline & BDI-II by trial type & -0.003 & 5.933 & 0.015 \\
\hline & RRS-R by BDI-II by trial type & 0.000 & 0.709 & 0.400 \\
\hline \multirow[t]{7}{*}{ Retrieval Beta RRS-R and BAI } & RRS-R & 0.010 & 0.565 & 0.452 \\
\hline & BAI & 0.008 & 1.081 & 0.300 \\
\hline & Trial type & $0.069^{*}$ & $8.929 *$ & $0.003 *$ \\
\hline & RRS-R by BAI & -0.001 & 0.006 & 0.937 \\
\hline & RRS-R by trial type & -0.005 & 1.958 & 0.162 \\
\hline & BAI by trial type & -0.001 & 4.770 & 0.029 \\
\hline & RRS-R by BAI by trial type & 0.001 & 2.436 & 0.119 \\
\hline \multirow[t]{7}{*}{ Retrieval Beta RRS-R and BDI-II } & RRS-R & 0.011 & 0.259 & 0.611 \\
\hline & BDI-II & 0.027 & 0.166 & 0.684 \\
\hline & Trial type & $0.093^{*}$ & $8.899 *$ & $0.003 *$ \\
\hline & RRS-R by BDI-II & -0.001 & 0.028 & 0.868 \\
\hline & RRS-R by trial type & -0.005 & 0.393 & 0.531 \\
\hline & BDI-II by trial type & -0.017 & 0.094 & 0.759 \\
\hline & RRS-R by BDI-II by trial type & 0.001 & 5.069 & 0.024 \\
\hline
\end{tabular}

Notes: Asterisk denotes significance at the 0.006 level. Ruminative Responses Scale-Revised (RRS-R), Beck Depression Inventory-II (BDI-II), Beck Anxiety Inventory (BAI), Old/hit/hit (OHH), Old/hit/miss (OHM), Old/hit/miss (OHM), b = beta

Significant interactions of nonclinical depression level and trait rumination were found for both old/hit/hit (Fig. 5A) and old/hit/miss or miss trials (Fig. 5B) during retrieval for alpha power, in which the relationship between depression and alpha power becomes more positive with increasing trait rumination. We predicted that during encoding and retrieval, alpha and beta power decreases post stimulus presentation would be less drastic for participants with higher trait rumination. This appears to be the case for alpha power, but mostly when rumination is interacting with depression. These changes in alpha and beta oscillatory power with trait anxiety, depression, and trait rumination may reflect differences in cognitive flexibility related to rumination leading to lessened memory for contextual details.

Individuals with higher trait rumination and higher levels of anxiety exhibit lessened memory for contextual details, which could be due to these individuals being more likely to get stuck on a thought (state rumination) during the task. Our results show that higher levels of trait anxiety and rumination predict an increase in the number of trials where a person forgets only the contextual detail associated with an item (Fig. 3B) and a decrease in the number of trials a person successfully remembers both the item and its associated contextual detail (Fig. 3A), showing that memory for contextual details associated with a test item is hindered. Because being able to recall contextual details related to a specific cue is an important feature of episodic memory, our results suggest episodic memory may not be optimally functioning in people that are highly ruminative when combined with higher trait levels of anxiety. Consistent with the cognitive inflexibility hypothesis of rumination (Altamirano, Mlyake, \& Whitmer, 2010; Davis, \& Nolen-Hoeksema, 2000; Koster, De Lissnyder, Derakshan, \& De Raedt, 2011; Nejad, Fossati, \& Lemogne, 2013; Yee lo, Lau, Cheung, \& Allen, 2012), a 


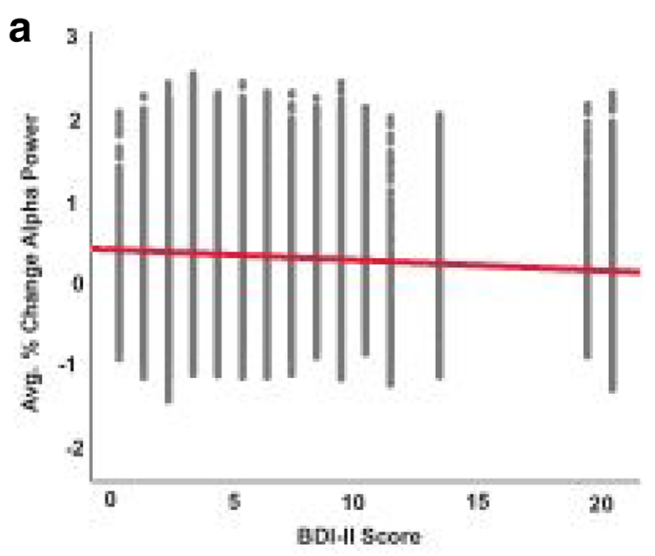

b

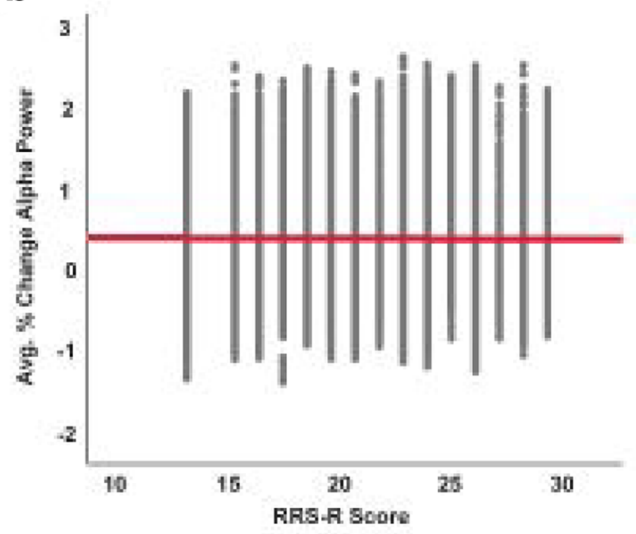

C

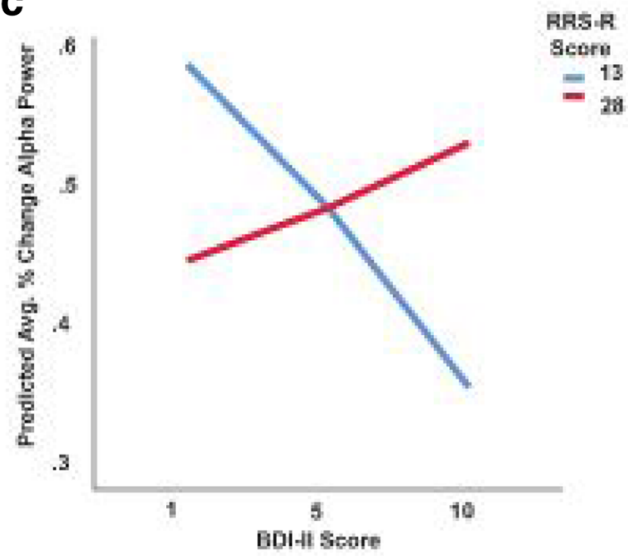

person high in trait rumination may get stuck on a thought and be unable to flexibly shift attention to focus on the encoding and/or retrieval of the details of an episode, which may lead to failure to retrieve a specific contextual detail. Individuals high in trait rumination express behaviors consistent with impaired cognitive flexibility (Altamirano, Mlyake, \& Whitmer, 2010; Davis, \& Nolen-Hoeksema, 2000; Whitmer \& Banich, 2007; Yee lo, Lau, Cheung, \& Allen, 2012). Whitmer and Banich
Fig. 4 ERSP results for alpha power during encoding. (A) Main effect of depression predicting percent change in alpha power during encoding for items that were later old/hit/hits. Each dot represents the percent change in alpha power from baseline for a single trial. Trials for all participants are included. The red line is the line of best fit to demonstrate the trend in percent change in alpha power as depression level increases. (B) Main effect of tendency to ruminate predicting percent change in alpha power during encoding for items that were later old/hit/hits. (C) The interaction of rumination and depression predicting the average percent change in alpha power during encoding for items that were later an old $/ \mathrm{hit} / \mathrm{miss}$ or miss. Scores were chosen for each variable and entered into the regression equation to generate predicted numbers for the average percent change in alpha power from baseline. For depression, the mean and one standard deviation away from the mean on either side were used for scores. For rumination, the highest (red line) and lowest (blue line) scores from our sample were used

(2007) demonstrated that individuals higher in trait rumination have trouble inhibiting prior mental sets, and they experience a higher cost for set switching. These results support the idea that participants with a higher tendency to ruminate combined with higher anxiety may have had troubles with getting mentally stuck during the study and/or test portion of the task, which in turn may impact the amount of detail encoded in a memory trace or details recalled during test. Ricarte et al. (2016) demonstrated that state anxiety enhances the association between trait rumination and episodic memory. Aspects of our task procedure may increase state anxiety, which in turn may enhance the association between trait rumination and memory functioning. Because memory decisions needed to be made for each item during test, more anxious participants with higher trait rumination may have been more likely to get stuck thinking about whether a previous decision was correct or more general anxious thoughts about their performance on the task, which may disrupt concentration on the task at hand leading to lessened memory for contextual details. Observing oscillatory power dynamics in the alpha band can help to determine whether cognitive inflexibility may be occurring during the task.

Cognitive inflexibility associated with trait rumination may lead to decreased memory for contextual details. Trait rumination alone is associated with higher alpha power (Fig. 4B) as is the interaction of rumination and nonclinical depression level (Figs. 4C, and 5A, B). Higher alpha power may be reflective of mental inflexibility based on the role that alpha power dynamics play in attention and the demonstrated relationship between alpha and trait rumination, which is characterized by decreased cognitive flexibility. Alpha power may work as a gating mechanism for neural resources in the brain so that brain areas important for the task at hand receive more neural resources than task irrelevant areas (Foxe et al., 2014; Foxe \& Snyder, 2011; Jensen \& Mazaheri, 2010; Klimesch, 2012; Moosmann et al., 2003; Serrien \& Sovijarvi-Spap, 2013). Thus, greater alpha power decreases within a specific brain region are associated with greater recruitment of neural 

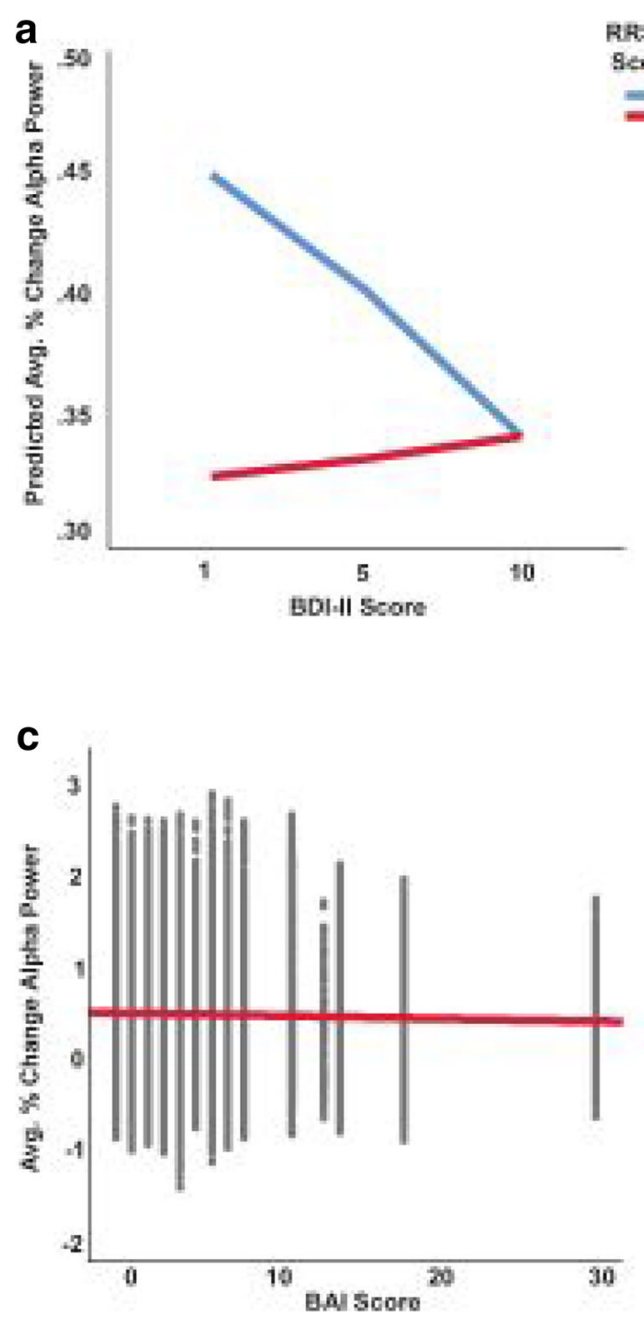

Fig. 5 ERSP results for alpha power during retrieval. (A) The interaction of rumination and depression when predicting the average percent change in alpha power during an old/hit/hit. Scores were chosen for each variable and entered in to the regression equation to generate predicted numbers for the average percent change in alpha power from baseline. For depression, the mean and one standard deviation away from the mean on either side were used for scores. For rumination, the highest (red line) and lowest (blue line) scores from our sample were used. (B) The interaction

resources, while higher power reflects recruitment of fewer neural resources (Jensen \& Mazaheri, 2010; Klimesch, 2012). In the current study, we demonstrate that as trait rumination increases, alpha power increases from baseline during encoding for trials that were later old/hit/hits (Fig. 4B). During encoding for trials that were later old/hit/misses or misses (Fig. 4C) and during retrieval for old/hit/hit (Fig. 5A) and old/hit/miss or miss trials (Fig. 5B), trait rumination interacts with nonclinical depression, the combination predicting higher alpha power as both depression and rumination increase. The increasing alpha power from baseline suggests less neural resources are being used in those with higher trait rumination combined with higher nonclinical depression level during encoding for items and/or details that are later forgotten
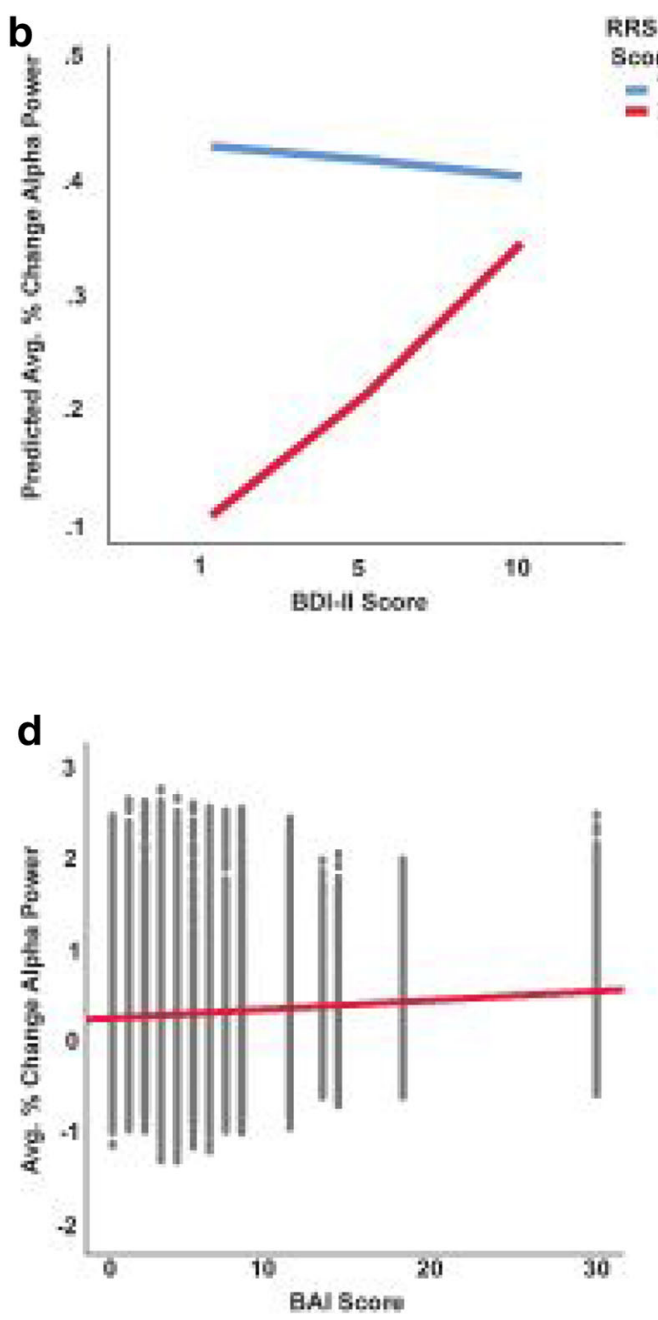

of rumination and depression when predicting the average percent change in alpha power during an old/hit/miss or miss. (C) Main effect of anxiety predicting percent change in alpha power during an old/hit/hit. Each dot represents the percent change in alpha power from baseline for a single trial. Trials for all participants are included. The red line is the line of best fit to demonstrate the trend in percent change in alpha power as anxiety level increases. (D) Main effect of anxiety predicting percent change in alpha power during an old/hit/miss or miss trial

and during retrieval in general. Less neural resource use in those with higher trait rumination and nonclinical depression levels could result in not enough resources being used to encode and/or retrieve specific contextual details. Individuals high in trait rumination may engage in state rumination and get stuck on other thoughts during encoding and/or retrieval, which could make fewer neural resources available when attempting to encode and/or retrieve the memory of an item and its contextual detail. This could help to explain the finding that trait rumination is a partial mediator in the relationship between depression and overgeneral autobiographical (Liu et al., 2017). Overgeneral autobiographical memory is characterized by a general lack of detail in autobiographical memory recall and reliance on schemas (Soderlund et al. 2014). 

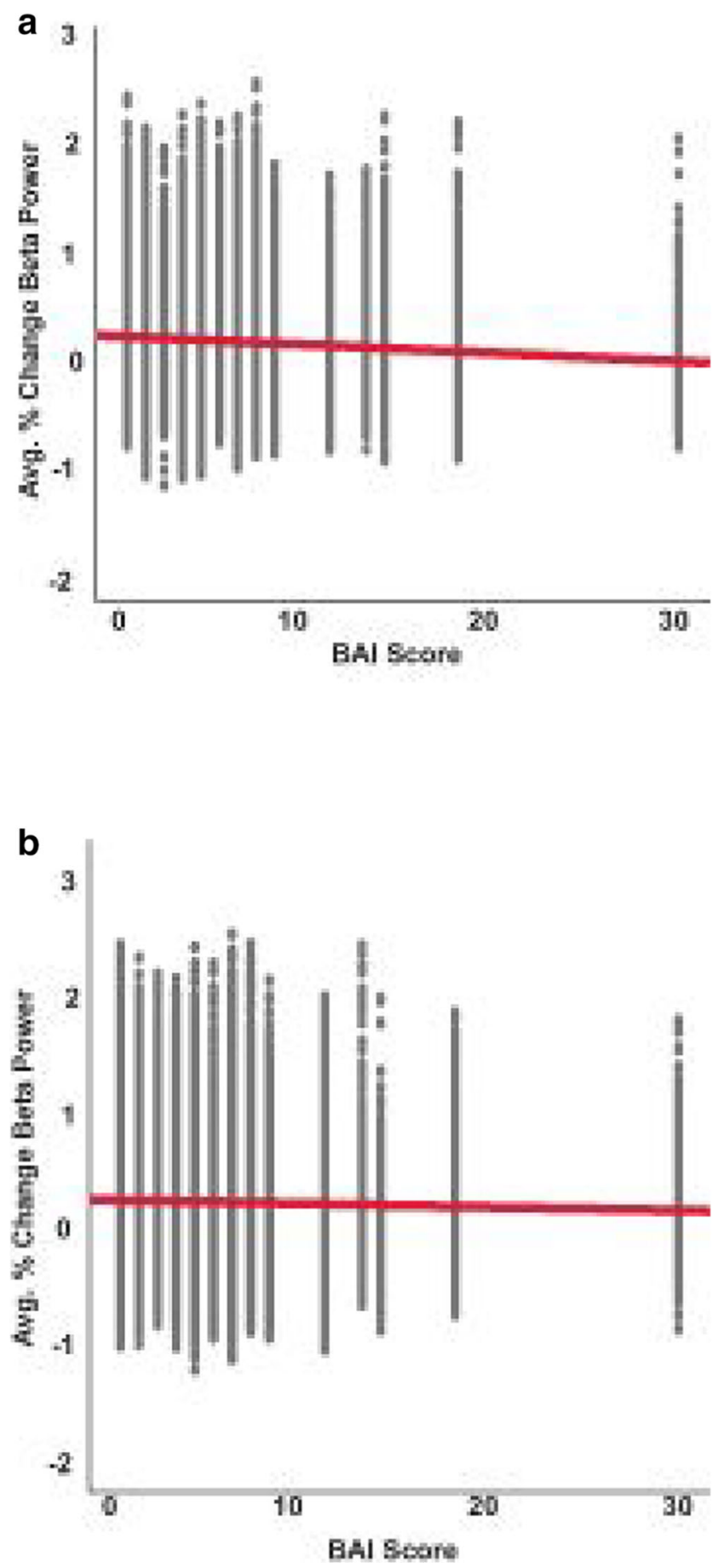

Fig. 6 ERSP results for beta power. Each dot represents the percent change in beta power from baseline for a single trial. Trials for all participants are included. The red line is the line of best fit to demonstrate the trend in percent change in beta power as anxiety level increases. (A) Main effect of anxiety predicting percent change in beta power during encoding for items that were later an old/hit/hit. (B) Main effect of anxiety predicting percent change in beta power during encoding for items that were later an old/hit/miss or miss

Based on our findings, rumination may decrease memory for specific contextual details. Reliance on more general schemas when recalling autobiographical memories may be a response to a decreased ability to remember specific contextual details. Rumination on depressive thoughts may be particularly difficult to redirect attention away from, especially for individuals diagnosed with major depressive disorder, which could exacerbate effects of rumination on episodic memory functioning (Koster et al., 2011). Failure to disengage from another thought leading to fewer neural resources available for the task at hand could explain why individuals with higher trait rumination paired with higher anxiety have more old/hit/miss trials (Fig. 3B) and fewer old/hit/hit trials (Fig. 3A). However, we did not have a measure of state rumination, so it is possible that participants higher in trait rumination may have had general attention difficulties rather than getting stuck on a thought. Future studies may be able to determine if state rumination is associated with higher alpha power and decreased memory for contextual details. Also, it is depression level interacting with trait rumination that predicts differences in alpha power, while it is trait anxiety interacting with rumination that predicts reduced memory for contextual details. Our results suggest that higher levels of nonclinical depression may increase the likelihood of getting stuck on a thought, but anxiety may play an additional role leading to difficulty with memory functioning.

Individuals higher in nonclinical depression level may be able to employ neural compensatory mechanisms to improve memory functioning. As depression alone increases, alpha power decreases more in posterior parietal regions for trials that were later old/hit/hits (Fig. 4A). Greater alpha power decreases are reflective of greater recruitment of neural resources (Jensen \& Mazaheri, 2010; Klimesch, 2012). It may be the case that the greater recruitment of neural resources reflects a compensatory mechanism for individuals with higher levels of depression. It may be harder for individuals higher in depression to focus their thoughts on the task at hand, and in order to focus properly, they recruit more neural resources. Krompinger and Simons (2011) demonstrated that during a verbal Stroop task, participants with high depression level showed larger P300 event-related potentials, which are associated with cognitive control resources (Huang, Chen, \& Zhang, 2015; Lu, Donamayor, Munte, \& Bahlmann, 2017; Polich, 2007) compared with participants lower in depression level. Behavioral performance on the task did not differ between the two groups, leading the authors to suggest that the greater recruitment of resources by the high depression group was a compensatory mechanism. In our study, as nonclinical depression level increases, alpha power decreases for items that were later old/hit/hits (Fig. 4A), which would support a compensatory mechanism. It could be the case that depression level on its own can be compensated for by increasing neural resource use, but when depression level is paired with tendency to ruminate, the increase in neural resource use in not enough to compensate for both, resulting in a decrease in memory ability. 
Trait anxiety may impact memory functioning due to its relationship with the control of attentional resources. During retrieval, higher trait anxiety is associated with greater alpha power decreases in posterior parietal areas during an old/hit/ hit (Fig. 5C), but power increases during an old/hit/miss (Fig. 5D). When attention is focused, alpha power increases in taskirrelevant brain areas and lowers in task-relevant areas leading to optimal attentional focus (Hanslmayr, Staudigl, \& Fellner, 2012; Jensen \& Mazaheri, 2010; Kizuk \& Mathewson, 2017; Klimesch, Doppelmayr, Russegger, Pachinger, \& Schwaiger, 1998; Klimesch, 2012; Minarik, Berger, \& Sauseng, 2018; Moorselaar et al., 2018; Vollebregt, Zumer, Huurne, Buitelaar, \& Jensen, 2016). Alpha power increases during an $\mathrm{old} / \mathrm{hit} / \mathrm{miss}$ trial associated with anxiety suggests that the participant's attention may be elsewhere when making a memory decision. Research shows that more anxious individuals have difficulty with attentional control (Eysenck \& Byrne, 1992; Eysenck, Derakshan, Santos, \& Calvo, 2007; Forster, NunezElizalde, Castle, \& Bishop, 2015; Reinholdt-Dunne \& Bradley, 2013). Thus, more anxious individuals may be less able to focus their attention on the task at hand to retrieve a specific contextual detail. Feedback on performance was not given to the participants; therefore, it is possible that more anxious individuals were distracted by anxious thoughts related to their performance on the task or more anxious thoughts in general (i.e., an upcoming exam, relationship with a friend/ partner, etc....). If anxious individuals are distracted by other thoughts, it could hinder their ability to recall specific contextual details.

More anxious thoughts and less attentional focus may lead to more complex memory traces being encoded during the task. Larger decreases in beta power may reflect a greater neural capacity to represent content-specific information contained in a memory (Hanslmayr et al., 2012, 2016; Khader \& Rösler, 2011; Staudigl et al., 2015; Waldhauser et al., 2016). Our analysis of beta power revealed that higher levels of trait anxiety are associated with greater power decreases in beta only during encoding (Fig. 6). This suggests that individuals higher in trait anxiety may be encoding more details when presented with a stimulus. However, the details that these individuals are encoding may not be directly related to the task at hand. More anxious individuals have difficulty with attentional control and may not be as able to narrow their attention to focus on encoding solely the item and the specific contextual detail. As such, they may encode other unrelated details of the experience, such as noises, smells, or any other thoughts they may have had during the task. Our behavioral findings show increasing trait anxiety and trait rumination predict less memory for contextual details (Fig. 3A and B). It could be the case that more anxious individuals encode details that are not task-relevant, which may then make it more difficult to remember the contextual detail required by the task. Future studies may be able to determine whether more anxious individuals encode details that are not task relevant. Explanations aside, the greater decreases in beta power with higher trait anxiety suggest the neural coding involved with the memory trace may have more memory content, but the memory trace may actually be hindering the retrieval of specific contextual details.

Combining our beta and alpha power results suggests that trait rumination, anxiety, and depression are associated with altered divisions of neural resources and neural coding of memory traces which may impact memory for contextual details. Our beta results suggest that more anxious individuals may be encoding more details, which could interfere with both encoding and recall of the specific contextual detail needed for the task. Results for alpha demonstrate that when trait rumination is paired with depression it may lead to hindered disengagement from other thoughts resulting in less recruitment of neural resources to focus on the task at hand. These two pieces of information combined may create a particularly difficult situation when it comes to recalling a specific contextual detail. The memory trace itself may not contain information about the specific contextual detail or the presence of other unrelated details may cause interference during retrieval. Higher alpha power and the use of less neural resources suggests that there may not be enough neural resources recruited to successfully combat any interference from other details and/or other thoughts and successfully recall the specific contextual detail. It should also be noted that the percent change in signal from baseline for alpha and beta power values were positively correlated $(r=0.65)$ in our study, but in memory studies it is not uncommon to find that alpha and beta oscillations show similar power dynamics (for review see Hanslmayr et al., 2016). Future studies can help untangle the different cognitive components that could be contributing to lessened memory for contextual details associated with trait rumination. In conclusion, when individuals with a higher tendency to ruminate are feeling more anxious, it impacts their episodic memory in a negative way, possibly due to the inability to flexibly shift and focus attention.

Acknowledgements This work was funded by the Psychology department at the University of New Hampshire. The authors thank Robert Drugan for providing feedback on the manuscript, Dr. Scott Slotnick for sharing his source memory task and stimuli with us, and Cameron Petit, Jessica Brown, Briana Silck, and Audrey Tocco for technical assistance. The authors declare no conflict of interest.

Open practices statement Deidentified behavior and raw EEG data are available on figshare.com. The behavior data can be accessed at the web address https://figshare.com/articles/Forner-Phillips_Mills_Ross_2020_ Source_Memory_Task_Behavior_Data/11879274. The EEG data can be accessed at the web address https://figshare.com/articles/FornerPhillips_Mills_Ross_2020_Source_Memory_Task_EEG_Data/ 11872110 . The authors are not at liberty to share the source memory task or the stimuli used because they are the property of Dr. Scott Slotnick. This experiment was not preregistered. 


\section{Appendix A}

Table 7. Results from the Correct Rejection proportion regression

\begin{tabular}{llll}
\hline & $\mathrm{b}$ & Standardized beta & $\mathrm{Sr}^{2}$ \\
\hline RRS-R & -0.006 & -0.170 & 0.023 \\
BDI-II & -0.002 & -0.069 & 0.002 \\
BAI & 0.004 & 0.127 & 0.003 \\
Interaction of RRS-R and BDI-II & 0.003 & 0.397 & 0.049 \\
Interaction of RRS-R and BAI & $-0.005^{*}$ & $-0.913^{*}$ & $0.158^{*}$ \\
Interaction of BDI-II and BAI & 0.001 & 0.242 & 0.010 \\
\hline
\end{tabular}

Notes: Asterisk denotes significance at the 0.05 level. Ruminative Responses Scale-Revised (RRS-R), Beck Depression Inventory-II (BDI-II), Beck Anxiety Inventory (BAI), b = beta, and $\mathrm{Sr}^{2}$ is the unique variance explained by a predictor variable

Table 8. Results from the False Alarm proportion regression

\begin{tabular}{llll}
\hline & $\mathrm{b}$ & Standardized beta & $\mathrm{Sr}^{2}$ \\
\hline RRS-R & 0.006 & 0.170 & 0.023 \\
BDI-II & 0.002 & 0.069 & 0.002 \\
BAI & -0.004 & -0.127 & 0.003 \\
Interaction of RRS-R and BDI-II & -0.003 & -0.397 & 0.049 \\
Interaction of RRS-R and BAI & $0.005^{*}$ & $0.913^{*}$ & $0.158^{*}$ \\
Interaction of BDI-II and BAI & -0.001 & -0.242 & 0.010 \\
\hline
\end{tabular}

Notes: Asterisk denotes significance at the .05 level. Ruminative Responses Scale-Revised (RRS-R), Beck Depression Inventory-II (BDI-II), Beck Anxiety Inventory (BAI), b = beta, and $\mathrm{Sr}^{2}$ is the unique variance explained by a predictor variable

Table 9. Results from $\mathrm{OHH}$ reaction time regression

\begin{tabular}{llll}
\hline & $\mathrm{b}$ & Standardized beta & $\mathrm{Sr}^{2}$ \\
\hline RRS-R & -0.036 & -0.242 & 0.047 \\
BDI-II & -0.010 & -0.068 & 0.002 \\
BAI & -0.006 & -0.055 & 0.001 \\
Interaction of RRS-R and BDI-II & 0.001 & 0.035 & 0.000 \\
Interaction of RRS-R and BAI & -0.014 & -0.593 & 0.067 \\
Interaction of BDI-II and BAI & 0.005 & 0.484 & 0.041
\end{tabular}

Notes: Asterisk denotes significance at the 0.05 level. Ruminative Responses Scale-Revised (RRS-R), Beck Depression Inventory-II (BDI-II), Beck Anxiety Inventory (BAI), b = beta, and $\mathrm{Sr}^{2}$ is the unique variance explained by a predictor variable

Table 10. Results from OHM reaction time regression

\begin{tabular}{llll}
\hline & $\mathrm{B}$ & Standardized beta & $\mathrm{Sr}^{2}$ \\
\hline RRS-R & -0.036 & -0.232 & 0.043 \\
BDI-II & -0.008 & -0.056 & 0.001 \\
BAI & -0.006 & -0.053 & 0.001 \\
Interaction of RRS-R and BDI-II & 0.001 & 0.017 & 0.000 \\
Interaction of RRS-R and BAI & -0.016 & -0.644 & 0.079 \\
Interaction of BDI-II and BAI & 0.005 & 0.491 & 0.042 \\
\hline
\end{tabular}

Notes: Asterisk denotes significance at the 0.05 level. Ruminative Responses Scale-Revised (RRS-R), Beck Depression Inventory-II (BDI-II), Beck Anxiety Inventory (BAI), b = beta, and $\mathrm{Sr}^{2}$ is the unique variance explained by a predictor variable
Table 11. Results from follow up linear regressions with alpha and beta power change as dependent variables

\begin{tabular}{lll}
\hline & $b$ & $p$ \\
\hline Alpha encoding BDI-II old/hit/hit & $-0.002^{*}$ & $<0.001^{*}$ \\
Alpha encoding RRS-R old/hit/hit & $0.008^{*}$ & $0.002^{*}$ \\
Alpha encoding BDI-II x RRS-R old/hit/hit & -0.001 & 0.080 \\
Alpha encoding BDI-II old/hit/miss+miss & $-0.048^{*}$ & $<0.001^{*}$ \\
Alpha encoding RRS-R old/hit/miss+miss & -0.010 & 0.978 \\
Alpha encoding BDI-II x RRS-R old/hit/miss+miss & $0.002^{*}$ & $<0.001^{*}$ \\
Beta encoding BAI old/hit/hit & $-0.008^{*}$ & $<0.001^{*}$ \\
Beta encoding BAI old/hit/miss+miss & $-0.003^{*}$ & $0.001^{*}$ \\
Alpha retrieval BDI-II old/hit/hit & -0.026 & 0.648 \\
Alpha retrieval RRS-R old/hit/hit & -0.010 & 0.070 \\
Alpha retrieval BDI-II x RRS-R old/hit/hit & $0.001^{*}$ & $<0.001^{*}$ \\
Alpha retrieval BDI-II old/hit/miss+miss & $-0.029^{*}$ & $<0.001^{*}$ \\
Alpha retrieval RRS-R old/hit/miss+miss & $-0.024^{*}$ & $<0.001^{*}$ \\
Alpha retrieval BDI-II x RRS-R old/hit/miss+miss & $0.002^{*}$ & $<0.001^{*}$ \\
Alpha retrieval BAI old/hit/hit & $-0.003^{*}$ & $0.005^{*}$ \\
Alpha retrieval BAI old/hit/miss+miss & $0.010^{*}$ & $<0.001^{*}$ \\
\hline
\end{tabular}

Notes: Asterisk denotes significance at the 0.006 level. Ruminative Responses Scale-Revised (RRS-R), Beck Depression Inventory-II (BDI-II), Beck Anxiety Inventory (BAI), b = beta

\section{References}

Altamirano, L.J., Miyake, A., \& Whitmer, A.J. (2010) When mental inflexibility facilitates executive control: Beneficial side effects of ruminative tendencies on goal maintenance. Psychological Science, 21(10), 1377-1382. https://doi.org/10.1177/0956797610381505

Army Individual Test Battery. (1944). Manual of Directions and Scoring. Washington, DC: War Department, Adjutant General's Office.

Baayen, R.H., Davidson, D.J., \& Bates, D.M. (2008). Mixed-effects modeling with crossed random effects for subjects and items. Journal of Memory and Language, 59(4), 390-412. https://doi.org/ 10.1016/j.jml.2007.12.005

Beck, A.T., Epstein, N., Brown, G., \& Steer, R.A., (1988). An inventory for measuring clinical anxiety: Psychometric properties. Journal of Consulting and Clinical Psychology, 56(6), 893-897. https://doi.org/ 10.1037/0022-006X.56.6.893

Beck, A.T., Steer, R.A., \& Brown, G.K., (1996) San Antonio, TX: Psychological Corporation

Bell, A. J. \& Sejnowski, T. J. (1995). An information-maximization approach to blind separation and blind deconvolution. Neural Computation, 7(6), 1129-1159. https://doi.org/10.1162/neco.1995. 7.6.1129

Bonnefond, M., Kastner, S., \& Jensen, O. (2017) Communication between brain areas based on nested oscillations. eNeuro, .0153-16. https://doi.org/10.1523/ENEURO.0153-16.2017

Buschman, T. J., Denovellis, E. L., Diogo, C., Bullock, D., \& Miller, E. K. (2012). Synchronous oscillatory neural ensembles for rules in the prefrontal cortex. Neuron, 76(4), 838-846. https://doi.org/10.1016/j. neuron.2012.09.029

Buschman, T. J., \& Miller, E. K. (2007) Top-down versus bottom-up control of attention in the prefrontal and posterior parietal cortices. Science, 315(5820), 1860-2. https://doi.org/10.1126/science. 1138071 
Clarke, A., Roberts, B. M. \& Ranganath, C. (2018). Neural oscillations during conditional associative learning. Neuroimage, 174, 485-493. https://doi.org/10.1016/j.neuroimage.2018.03.053

Cooper, P. S., Darriba, A., Karayanidis, F., \& Barcelo, F. (2016). Contextually sensitive power changes across multiple frequency bands underpin cognitive control. NeuroImage, 132, 22-511. https://doi.org/10.1016/j.neuroimage.2016.03.010

Cunillera, T., Fuentemilla, L., Perianez, J., Marco-Pallares, J., Kramer, U. M., Camara, E., Munte, T. F., \& Rodriguiez-Fornells, A. (2012). Brain oscillatory activity associated with task switching and feedback processing. Cognitive, Affective, and Behavioral Neuroscience, 12(1), 16-33. https://doi.org/10.3758/s13415-011$0075-5$

Davis, R.N., \& Nolen-Hoeksema, S. (2000) Cognitive inflexibility among ruminators and nonruminators. Cognitive Therapy and Research, 25(6), 699-711. https://doi.org/10.1023/A: 1005591412406

Delorme, A. \& Makeig, S. (2004). EEGLAB: an open source toolbox for analysis of single-trial EEG dynamics including independent component analysis. Journal of Neuroscience Methods, 134(1), 9-21. https://doi.org/10.1016/j.jneumeth.2003.10.009

Düzel, E., Habib, R., Schott, B., Schoenfeld, A., Lobaugh, N., Mcintosh, A.R., Scholz, M., Heinze, H.J. (2003) A multivariate, spatiotemporal analysis of electromagnetic time-frequency data of recognition memory. Neuroimage, 18(2), 185-197. https://doi.org/10.1016/ S1053-8119(02)00031-9

Engel, A.K., \& Fries, P. (2010). Beta-band oscillations - signaling the status quo? Current Opinion in Neurobiology, 20, 156-165. https:// doi.org/10.1016/j.conb.2010.02.015

Eysenck, M.W., \& Byrne, A. (1992). Anxiety and susceptibility to distraction. Personality and Individual Differences, 13, 793-798. https://doi.org/10.1016/0191-8869(92)90052-Q

Eysenck, M.W., Derakshan, N., Santos, R., \& Calvo, M.G. (2007). Anxiety and Cognitive Performance: Attentional Control Theory. Emotion, 7(2), 336-353. https://doi.org/10.1037/1528-3542.7.2.336

Ferdek, M.A., Van Rijn, C.M., \& Wyczesany M. (2016). Depressive rumination and the emotional control circuit: An EEG localization and effective connectivity study. Cognitive, Affective, and Behavioral Neuroscience, 16, 1099-1113. https://doi.org/10.3758/ s13415-016-0456-x

Forster, S., Nunze-Elizalde, A.O., Castle, E., \& Bishop, S.J. (2015). Unraveling the Anxious Mind: Anxiety, Worry, and Frontal Engagement in Sustained Attention Versus Off-Task Processing. Cerebral Cortex, 25(3), 609-618. https://doi.org/10.1093/cercor/ bht 248

Foxe, J. J., Murphy, J. W., \& Sanctis, P. D. (2014). Throwing out the rules: anticipatory alpha band oscillatory attention mechanisms during task-set reconfigurations. European Journal of Neuroscience, 39, 1960-1972. https://doi.org/10.1111/ejn.12577

Foxe, J. J., \& Snyder, A. C. (2011). The role of alpha-band oscillations as a sensory suppression mechanism during selective attention. Frontiers in Psychology, 2. https://doi.org/10.3389/fpsyg.2011. 00154

Grandchamp, R., \& Delorme, A. (2011) Single-trial normalization for event-related spectral decomposition reduces sensitivity to noisy trials. Frontiers in Psychology, 2, 1-14. https://doi.org/10.3389/ fpsyg.2011.00236

Haegens, S., Vergara, J., Rossi-Pool, R., Lemus, L., \& Romo, R. (2017). Beta oscillations reflect supramodal information during perceptual judgment. pNAS, 114(52), 13810-13815. https://doi.org/10.1073/ pnas. 1714633115

Hanslmayr, S., Leipold, P., Pastötter, B. \& Bäuml, K. H. (2009a). Anticipatory signatures of voluntary memory suppression. The Journal of Neuroscience: The Official Journal for the Society for Neuroscience, 29(9), 2742-2747. https://doi.org/10.1523/ JNEUROSCI.4703-08.2009
Hanslmayr, S., Matuschek, J., \& Fellner, M.C. (2014). Entrainment of Prefrontal Beta Oscillations Induces an Endogenous Echo and Impairs Memory Formation. Current Biology, 24, 904-909. https:// doi.org/10.1016/j.cub.2014.03.007

Hanslmayr, S., Spitzer, B., \& Bauml, K. (2009b). Brain oscillations dissociate between semantic and nonsemantic encoding of episodic memories. Cerebral Cortex, 19, 1631-1640. https://doi.org/10. 1093/cercor/bhn197

Hanslmayr, S., Staresina, B. P. \& Bowman, H. (2016). Oscillations and episodic memory: Addressing the synchronization/ desynchronization conundrum. Trends in Neurosciences, 39(1),1625. https://doi.org/10.1016/j.tins.2015.11.004

Hanslmayr, S., Staudigl, T. \& Fellner, M. C. (2012). Oscillatory power decreases and long-term memory: the information via desynchronization hypothesis. Frontiers in Human Neuroscience, 6, 74. https://doi.org/10.3389/fnhum.2012.00074

Huang, W.J., Chen, W.W., \& Zhang, X. (2015). The neurophysiology of P 300 - an integrated review. European Review for Medical and Pharmacological Sciences, 19(8), 1480-1488.

Jafarpour, A., Fuentemilla, L., Horner, A.J., Penny, W., \& Duzel, E. (2014). Replay of Very Early Encoding Representations during Recollection. The Journal of Neuroscience, 34(1), 242-248. https:// doi.org/10.1523/JNEUROSCI.1865-13.2014

Jensen, O. \& Mazaheri, A. (2010). Shaping functional architecture by oscillatory alpha activity: Gating by inhibition. Frontiers in Human Neuroscience, 4, 186. https://doi.org/10.3389/fnhum.2010.00186

Khader, P. H. \& Rösler, F. (2011). EEG power changes reflect distinct mechanisms during long-term memory retrieval. Psychophysiology, 48(3), 362-369. https://doi.org/10.1111/j.1469-8986.2010.01063.x

Kizuk, S.A., \& Mathewson, K.E. (2017). Power and Phase of Alpha Oscillations Reveal an Interaction between Spatial and Temporal Visual Attention. Journal of Cognitive Neuroscience, 29(3), 480494. https://doi.org/10.1162/jocn a 01058

Klimesch, W. (2012). Alpha-band oscillations, attention, and controlled access to stored information. Trends in Cognitive Sciences, 16(12), 606-617. https://doi.org/10.1016/j.tics.2012.10.007

Klimesch, W., Doppelmayr, M., Schimke, H. \& Ripper, B. (1997). Theta synchronization and alpha desynchronization in a memory task. Psychophysiology 34(2), 169-176. https://doi.org/10.1111/j.14698986.1997.tb02128.x

Klimesch, W., Doppelmayr, M., Russegger, H., Pachinger, T., \& Schwaiger, J. (1998). Induced alpha band power changes in the human EEG and attention. Neuroscience Letters, 244, 73-76. https://doi.org/10.1016/S0304-3940(98)00122-0

Klimesch, W., Schimke, H., Doppelmayr, M., Ripper, B., Schwaiger, J. \& Pfurtscheller, G. (1996) Event-related desynchronization (ERD) and the Dm effect: does alpha desynchronization during encoding predict later recall performance? International Journal of Psychophysiology: Official Journal of the International Organization of Psychophysiology, 24(1), 47-60. https://doi.org/ 10.1016/S0167-8760(96)00054-2

Koster, E.H.W., De Lissnyder, E., Derakshan, N., \& De Raedt, R. (2011). Understanding depressive rumination from a cognitive science perspective: The impaired disengagement hypothesis. Clinical Psychology Review, 31(1), 138-145. https://doi.org/10.1016/j.cpr. 2010.08.005

Krompinger, J.W., \& Simons, R.F. (2011). Cognitive inefficiency in depressive undergraduates: Stroop processing and ERPs. Biological Psychology, 86, 239-246. https://doi.org/10.1016/j. biopsycho.2010.12.004

Liu, X., Li, L., Xiao, J., Yang, J., \& Jiang, X. (2013). Abnormalities of autobiographical memory of patients with depressive disorders: A meta-analysis. Psychology and Psychotherapy, 86(4), 353-373. https://doi.org/10.1111/j.2044-8341.2012.02077.x

Liu, Y., Yu, X., Yang, B., Zhang, F., Zou, W., Na, A., Zhao, X., \& Yin, G. (2017). Rumination mediates the relationship between 
overgeneral autobiographical memory and depression in patients with major depressive disorder. BMC Psychiatry, 17, 103. https:// doi.org/10.1186/s12888-017-1264-8

Lu, M., Donamayor, N., Munte, T.F., \& Bahlmann, J. (2017). Eventrelated potentials and neural oscillations dissociate levels of cognitive control. Behavioural Brain Research, 320, 154-164. https://doi. org/10.1016/j.bbr.2016.12.012

Meconi, F., Anderl-Straub, S., Raum, H., Landgrebe, M., Langguth, B., Bauml, K., \& Hanslmayr, S. (2016). Aberrant prefrontal beta oscillations predict episodic memory encoding deficits in schizophrenia. NeuroImage: Clinical, 12, 499-505. https://doi.org/10.1016/j.nicl. 2016.08.017

Minarik, T., Berger, B. \& Sauseng, P. (2018). The involvement of alpha oscillations in voluntary attention directed towards encoding episodic memories. Neuroimage, 166, 307-316. https://doi.org/10.1016/j. neuroimage.2017.10.064

Mognon, A., Jovicich, J., Bruzzone, L., \& Buiatti, M. (2011). ADJUST: An automatic EEG artifact detector based on the joint use of spatial and temporal features. Psychophysiology, 48, 229-240. https://doi. org/10.1111/j.1469-8986.2010.01061.x

Mölle, M., Marshall, L., Fehm, H.L. \& Born, J. (2002). EEG theta synchronization conjoined with alpha desynchronization indicate intentional encoding. The European Journal of Neuroscience, 15(5), 923 928. https://doi.org/10.1046/j.1460-9568.2002.01921.x

Moorselaar, D., Foster, J.J., Sutterer, D.W., Theeuwes, J., Olivers, C.N.L., \& Awh, E. (2018). Spatially selective alpha oscillations reveal moment by moment trade-offs between working memory and attention. Journal of Cognitive Neuroscience, 30(2), 256-266. https://doi.org/10.1162/jocn_a 01198

Moosmann, M., Ritter, P., Krastel, I., Brink, A., Thees, S., Blankenburg, F., Taskin, B., Obrig, H., \& Villringer, A. (2003). Correlates of alpha rhythm in functional magnetic resonance imaging and near infrared spectroscopy. Neuroimage, 20, 145-158. https://doi.org/ 10.1016/S1053-8119(03)00344-6

Murphy, V., \& Dunne, A. (2005). Mixed effects versus fixed effects modelling of binary data with inter-subject variability. Journal of Pharmacokinetics and Pharmacodynamics, 32(2), 245-260. https:// doi.org/10.1007/s10928-005-0045-Z

Nejad, A.B., Fossati, P., \& Lemogne, C. (2013). Self-referential processing, rumination, and cortical midline structures in major depression. Frontiers in Human Neuroscience, 7, 666. https://doi.org/10.3389/ fnhum.2013.00666

Noh, E., Herzmann, G., Curran, T., \& De Sa, V.R. (2014). Using singletrial EEG to predict and analyze subsequent memory. NeuroImage, 84, 712-723. https://doi.org/10.1016/j.neuroimage.2013.09.028

Nolen-Hoeksema, S., Wisco, B.E., \& Lyubomirsky, S. (2008) Rethinking rumination. Perspectives on Psychological Science, 3(5), 400-424. https://doi.org/10.1111/j.1745-6924.2008.00088.x

Nyhus, E. (2018). Brain Networks Related to Beta Oscillatory Activity during Episodic Memory Retrieval. Journal of Cognitive Neuroscience, 30(2), 174-187. https://doi.org/10.1162/jocn_a_ 01194

Oswal, A., Litvak, V., Brucke, C., Huebl, J., Schneider, G., Kuhn, A. A., \& Brown, P. (2012). Cognitive factors modulate activity within the human subthalamic nucleus during voluntary movement in Parkinson's Disease. Journal of Neuroscience, 33(40), 1581515826. https://doi.org/10.1523/JNEUROSCI.1790-13.2013

Parish, G., Hanslmayr, S. \& Bowman, H. (2018). The Sync/deSync Model: How a synchronized hippocampus and a desynchronized neocortex code memories. The Journal of Neuroscience: The Official Journal for the Society for Neuroscience, 38(14), 34283440. https://doi.org/10.1523/JNEUROSCI.2561-17.2018

Pearson (2001). Wechsler test of adult reading. San Antonio, TX: Pearson Education.

Phillips, J.M., Vinck, M., Everling, S., Womelsdorf, T. (2014). A longrange fronto-parietal 5-to $126-\mathrm{Hz}$ network predicts "top down" controlled guidance in a task-switch paradigm. Cerebral Cortex, 24, 1996-2008. https://doi.org/10.1093/cercor/bht050

Polich, J. (2007). Updating P300: an integrative theory of P3a and P3b. Clinical Neurophysiology, 118(10), 2128-2148. https://doi.org/10. 1016/j.clinph.2007.04.019

Putnam, K.M., \& McSweeney, L.B. (2008). Depressive symptoms and baseline prefrontal EEG alpha activity: A study utilizing ecological momentary assessment. Biological Psychology, 77, 237-240. https://doi.org/10.1016/j.biopsycho.2007.10.010

Reinholdt-Dunne, M.L., \& Bradley, B.P. (2013). Attention control: relationships between self-report and behavioural measures, and symptoms of anxiety and depression. Cognition \& Emotion, 27(3), 430440. https://doi.org/10.1080/02699931.2012.715081

Ricarte, J.J., Ros, L., Latorre, J.M., Munoz, M.D., Aguilar, M.J., \& Hernandez, J.V. (2016). Role of anxiety and brooding in specificity of autobiographical recall. Scandinavian Journal of Psychology, 57, 495-500. https://doi.org/10.1111/sjop.12323

Ross, R.S., \& Slotnick, S.D. (2008). The hippocampus is preferentially associated with memory for spatial context. Journal of Cognitive Neuroscience, 20(3), 432-446. https://doi.org/10.1162/jocn.2008. 20035

Schmitz, F., \& Voss, A. (2012). Decomposing task-switching costs with the diffusion model. Journal of Experimental Psychology: Human Perception and Performance, 38(1), 222-250. https://doi.org/10. 1037/a0026003

Serrien, D., \& Sovijarvi-Spape, M. M. (2013). Cognitive control of response inhibition and switching: Hemispheric lateralization and hand preference. Brain and Cognition, 82, 283-290. https://doi.org/ 10.1016/j.bandc.2013.04.013

Slotnick, S.D., \& Schacter, D.L. (2004). A sensory signature that distinguishes true from false memories. Nature Neuroscience, 7, 664-672. https://doi.org/10.1038/nn1252

Soderlund, H., Moscovitch, M., Kumar, N., Daskalakis, Z., Flint, A., Herrmann, N., \& Levine, B. (2014) Autobiographical episodic memory in major depressive disorder. Journal of Abnormal Psychology, 123(1), 51-60. https://doi.org/10.1037/a0035610

Spitzer, B., \& Haegens, S. (2017). Beyond the Status Quo: A Role for Beta Oscillations in Endogenous Content (Re)Activation. eNeuro, 4(4), 1-15. https://doi.org/10.1523/ENEURO.0170-17.2017

Staudigl, T., Vollmar, C., Noachtar, S., \& Hanslmayr, S. (2015). Temporal-pattern similarity analysis reveals the beneficial and detrimental effects of context reinstatement on human memory. The Journal of Neuroscience, 35(13), 5373-5384. https://doi.org/10. 1523/JNEUROSCI.4198-14.2015

Treynor, W., Gonzalez, R., \& Nolen-Hoeksema, S. (2003). Rumination reconsidered: A psychometric analysis. Cognitive Therapy and Research, 27(3), 247-259. https://doi.org/10.1023/A: 1023910315561

Thut, G., Nietzel, A., Brandt, S. A., Pascual-Leone, A. (2006). $\alpha$-band electroencephalographic activity over occipital cortex indexes visuospatial attention bias and predicts visual target detection. Journal of Neuroscience, 26(37), 9494-9502. https://doi.org/10. 1523/JNEUROSCI.0875-06.2006

Van Driel, J., Gunseli, E., Meeter, M., \& Olivers, C.N.L. (2017). Local and interregional alpha EEG dynamics dissociate between memory for search and memory for recognition. Neurolmage, 149, 114-128. https://doi.org/10.1016/j.neuroimage.2017.01.031

Waldhauser, G. T., Braun, V. \& Hanslmayr, S. (2016). Episodic Memory Retrieval Functionally Relies on Very Rapid Reactivation of Sensory Information. The Journal of Neuroscience: The Official Journal for the Society for Neuroscience, 36(1), 251-260. https:// doi.org/10.1523/JNEUROSCI.2101-15.2016

Wechsler, D. A. (1981). Wechsler Adult Intelligence Scale-Revised. New York: Psychological Corporation. 
Whitmer, A.J., \& Banich, M.T. (2007). Inhibition versus switching deficits in different forms of rumination. Psychological Science, 18(6), 546-553. https://doi.org/10.1111/j.1467-9280.2007.01936.x

Wolff, N., Zink, N., Stock, A., \& Beste, C. (2017). On the relevance of the alpha frequency oscillation's small-world network architecture for cognitive flexibility. Scientific Reports, 7, 13910. https://doi.org/ 10.1038/s41598-017-14490-x

Worden, M. S., Foxe, J. J., Wang, N., \& Simpson, G. V. (2000). Anticipatory biasing of visuospatial attention indexed by retinotopically specific $\alpha$-band electroencephalography increases over occipital cortex. Journal of Neuroscience, 20. https://doi.org/ 10.1523/JNEUROSCI.20-06-j0002.2000
Vollebregt, M.A., Zumer, J.M., Huurne, N., Buitelaar, J.K., \& Jensen, O. (2016). Posterior alpha oscillations reflect attentional problems in boys with Attention Deficit Hyperactivity Disorder. Clinical Neurophysiology, 127, 2182-2191. https://doi.org/10.1016/j. clinph.2016.01.021

Yee Lo, B.C., Lau, S., Cheung, S., \& Allen, N.B. (2012). The impact of rumination on internal attention switching. Cognition and Emotion, 26(2), 209-223. https://doi.org/10.1080/02699931.2011.574997

Publisher's note Springer Nature remains neutral with regard to jurisdictional claims in published maps and institutional affiliations. 